\title{
On Pose Estimation in Room-Scaled Environments
}

\author{
Hanna E. Nyqvist
}


This is a Swedish Licentiate's Thesis.

Swedish postgraduate education leads to a Doctor's degree and/or a Licentiate's degree.

A Doctor's Degree comprises 240 ECTS credits (4 years of full-time studies).

A Licentiate's degree comprises 120 ECTS credits,

of which at least 60 ECTS credits constitute a Licentiate's thesis.

Linköping studies in science and technology. Thesis

No. 1765

On Pose Estimation in Room-Scaled Environments

Hanna E. Nyqvist

\author{
hanna.nyqvisteliu.se \\ ww. control.isy.liu.se \\ Department of Electrical Engineering \\ Linköping University \\ SE-581 83 Linköping \\ Sweden
}

ISBN 978-91-7685-628-4

ISSN 0280-7971

Copyright (C) 2016 Hanna E. Nyqvist

Printed by LiU-Tryck, Linköping, Sweden 2016 
Systra mi, du är bäst! Ikväll ska vi tokdansa med Ronny och Ragge på bordet tills byxorna spricker så att mamma får dåndimpen och pappa ramlar ur soffan. 



\section{Abstract}

Pose (position and orientation) tracking in room-scaled environments is an enabling technique for many applications. Today, virtual reality (VR) and augmented reality $(\mathrm{AR})$ are two examples of such applications, receiving high interest both from the public and the research community. Accurate pose tracking of the VR or AR equipment, often a camera or a headset, or of different body parts is crucial to trick the human brain and make the virtual experience realistic. Pose tracking in room-scaled environments is also needed for reference tracking and metrology. This thesis focuses on an application to metrology. In this application, photometric models of a photo studio are needed to perform realistic scene reconstruction and image synthesis. Pose tracking of a dedicated sensor enables creation of these photometric models. The demands on the tracking system used in this application is high. It must be able to provide sub-centimeter and sub-degree accuracy and at same time be easy to move and install in new photo studios.

The focus of this thesis is to investigate and develop methods for a pose tracking system that satisfies the requirements of the intended metrology application. The Bayesian filtering framework is suggested because of its firm theoretical foundation in informatics and because it enables straightforward fusion of measurements from several sensors. Sensor fusion is in this thesis seen as a way to exploit complementary characteristics of different sensors to increase tracking accuracy and robustness. Four different types of measurements are considered; inertialmeasurements, images from a camera, range (time-of-flight) measurements from ultra wide band (UWB) radio signals, and range and velocity measurements from echoes of transmitted acoustic signals.

A simulation study and a study of the Cramér-Rao lower filtering bound (CRLB) show that an inertial-camera system has the potential to reach the required tracking accuracy. It is however assumed that known fiducial markers, that can be detected and recognized in images, are deployed in the environment. The study shows that many markers are required. This makes the solution more of a stationary solution and the mobility requirement is not fulfilled. A simultaneous localization and mapping (SLAM) solution, where naturally occurring features are used instead of known markers, are suggested solve this problem. Evaluation using real data shows that the provided inertial-camera SLAM filter suffers from drift but that support from UWB range measurements eliminates this drift. The SLAM solution is then only dependent on knowing the position of very few stationary UWB transmitters compared to a large number of known fiducial markers. As a last step, to increase the accuracy of the SLAM filter, it is investigated if and how range measurements can be complemented with velocity measurement obtained as a result of the Doppler effect. Especially, focus is put on analyzing the correlation between the range and velocity measurements and the implications this correlation has for filtering. The investigation is done in a theoretical study of reflected known signals (compare with radar and sonar) where the CRLB is used as an analyzing tool. The theory is validated on real data from acoustic echoes in an indoor environment. 



\section{Populärvetenskaplig sammanfattning}

Skattningar av position och åt vilket håll något är riktat (orienterat) behövs inom många olika områden. Virtuell verklighet (virtual reality) samt utökad eller förstärk verklighet (augmented reality) är två exempel på sådana områden som är väldigt aktuella idag både för allmänheten och inom flera olika forskningfält. Begreppet virtuel verklighet innebär en teknik som gör det möjligt att uppleva och verka i påhittade världar på ett sätt som känns verkligt. Begreppet förstärkt verklighet har liknande innebörd. Det innebär att vi människor ska kunna se, uppleva och interagera med påhittade ting och föremål som har stoppats in i vår egen fysiska värld. För att dessa påhittade verkligheter ska kännas realistiska så måste man kunna skatta position och orientering av exempelvis den utrustning som används, ofta någon typ av kamera, eller olika kroppsdelar, exempelvis åt vilket håll man tittar.

Ett annat exempel då skattning av position och orientering behövs är inom projektet Virtuel Photo Set (VPS), virtuell fotostudio. I detta projekt vill man utveckla metoder för att kunna datorgenerera fotorealistiska bilder av olika omgivningar där man kan lägga till påhittade föemål som inte fanns på plats när ursprungsbilderna togs. Detta kan vara bra om man exempelvis vill möblera om i sitt hus och vill se vilken soffa som passar bäst innan man köper någon. Vid skapande av bilder på detta sätt behövs bra modeller över hur omgivningarna ser ut. För att kunna skapa dessa modeller behöver man skatta positionen och orienteringen av den sensor som används för att mäta upp modellerna. Noggrannheten på positions- och orienteringsskattningarna måste vara hög, i storleksordning av millimetrar för positionen och delar av en grad för orienteringen. Dessutom måste skattningssystemet vara enkelt att flytta till en ny fotostudio så fasta installationer bör användas i så liten utsträckning som möjligt.

VPS-projektet har en central del i denna avhandling. Målet med avhandlingen är att undersöka och utveckla olika metoder för att kunna uppnå de krav på skattning av position och orientering som ställs i vPS-projektet. För att kunna uppnå detta behövs det sensorer som kan mäta och ge information om den sanna positionen och orienteringen. Det finns många olika typer av sensorer som kan bidra med värdefull information. Vissa kräver fasta installationer och andra inte. System med fasta installationer är oftast mer tillförlitliga och noggranna, speciellt om de används under en längre tid, men att behöva utföra installationen i varje ny fotostudio är tidskrävande.

I avhandlingen föreslås att man inte ska nöja sig med bara en typ av sensor utan att man ska utnyttja flera olika typer av sensorer. Då kan man utnyttja att de olika sensorerna kan komplettera varandras svagheter. Ett ramverk för hur man kan använda flera olika sensortyper presenteras och olika typer av sensorer och sensorkombinationer utvärderas. Det undersöks också hur man kan utvinna så mycket information som möjligt ur de mätningar man får, så att man inte kastar bort värdefull information. För sensorer som använder sig av fasta installationer så beror informationsmängden i mätningarna på hur de olika delarna av installa- 
tionen placerats ut. Detta innebär att man genom att planera bättre i förväg kan påverka så att en sensor bidrar med mer information i varje mätning. På så vis kan man få bättre skattningar av position och orientering.

Den första sensorn som används i denna avhandling är en IMU. Den mäter acceleration och hur snabbt något roterar. Med dessa mätningar kan man, genom att veta hur länge och hur fort något har rört sig, räkna ut både position och orientering för ett föremål. IMU:n är en sensor som inte behöver några fasta installationer och som kan skatta snabba korta förlopp väldigt bra men tyvärr så ackumuleras skattningsfelen över tiden och man får mindre noggranna skattningar ju längre tiden går.

Den andra sensorn som används är en kamera. Med hjälp av den kan man hitta och känna igen kända föremål i bilder och räkna ut vilken position och orientering man har i förhållande till dessa kända föremål. Ofta används kända mönsterbilder som placeras ut, men det går även att använda sig av saker som finns naturligt i omgivningarna. I avhandlingen testas båda dessa metoder. Mönsterbilderna kräver en installation men ger noggrannare resultat. Att behandla de bilder man får från kameran och hitta och känna igen alla föremål är beräkningskrävande och tar längre tid än att behandla mätningar från exempelvis en IMU. Kameran är därför inte lika bra som IMU:n på att hantera snabba förlopp. Med en kamera kan det dessutom vara svårt att skatta avstånd eftersom vår tredimensionella värld har projicerats ner till en tvådimensionell bild.

Den tredje sensorn som används är en radiomottagare. Med hjälp av denna kan man ta emot signaler från radiosändare som sitter installerade i omgivningarna. Genom att mäta hur lång tid det tar att skicka en signal från sändaren till mottagaren kan man räkna ut hur långt ifrån varandra dessa är. En radiomottagare behöver fast installation av radiosändare och kan inte skatta orientering, men man kan få noggranna avståndsskattningar och om man kombinerar mätningar från flera sändare kan man även få noggranna positionsskattningar.

När exempelvis en radio-, ljus-, eller ljudskignal skickas mellan två förmål kan man få information om avståndet så som beskrivits ovan. Signalen påverkas dock också av dopplereffekten. Dopplereffekten gör till exempel att sirenen från en ambulans låter annorlunda beroende på hur fort ambulansen kör och om den kör mot dig eller från dig. Tack vare denna effekt kan man kan få hastighetsinformation, inte bara avståndsinformation, från signaler som skickats mellan två platser. Det är vanligt att använda sådana kombinerade avstånds- och hastighetsmätningar utomhus. Exempelvis radarsensorer använder denna principen. Det är dock inte vanligt att denna typ av avstånds- och hastighetsmätningar används inomhus. I denna avhandling undersöks därför om och hur man kan utnyttja detta även inomhus.

I avhandlingen visas att man, genom att kombinera dessa olika typer av sensorer, kan bygga ett skattningssystem som är noggrannt både för korta förlopp och för långa och som endast kräver fast installation av ett mycket fåtal antal komponenter. 


\section{Acknowledgments}

First of all I would like to thank the two people that have helped me most with my work during these first years as a PhD student. Behind every successful PhD student there are a couple of exhausted supervisors, in my case Gustaf Hendeby and Fredrik Gustafsson. Thank you for pushing me when needed, holding me back when needed and being there to help day as night, weekday as weekend. I hope I have learned enough by now to make your work easier in the future.

Second of all I would like to thank the people helping me with this thesis. Thank you Clas Veibäck for being my precursor with the new template and cover fixing thing so that I wouldn't have to. Thank you Rickard Karlsson, Manon Kok, Martin Skoglund, Gustaf Hendeby, Fredrik Gustafsson and dad. Your comments have been very thorough and thought through and have helped me a lot. Believe it or not, but thank you also to my mum. Even though you don't even understand the title of my thesis you were able to help ;)

Special thanks to my dad also for always being the first one to ask to read my papers. Even though I usually sigh when you ask, I secretly like it. Getting your comments and your approval pleases my heart.

Per, I have told my friends that I would not mention you in my acknowledgement. I have said that I don't like when things get too emotional, that I would stick to facts and thank only the people whose support have had a direct impact on my thesis. The response from my friends was harsh... I am a cold hearted person that don't know how to show appreciation they said. Therefore I have found a way around this, to thank you but still keep to the facts. So, Per I thank you for being by my side. Without you I would have had to split my time between research and picking up boys. Then this thesis still wouldn't have been finished! (Thank you also for the weekend right before my first test print. You were cooking for me and taking care of me very sweetly. I hope we can repeat that many times :D)

I also want to thank all of my colleagues at RT. I think our group is amazing and we should be proud of and cherish what we have. Special thanks to my fellow PhD students. I don't want to call you colleagues, to me you are my friends. I have so many nice memories with you; hotel breakfasts; beer evenings; parties; film education (some evenings more frightening than others); bike tours; BBQ:s (both official and in-official); adventurous hiking/biking/skiing/climbing/canoeing trips with talking shoes, broken ribs, twisted chains, amazing fires, dancing polar bears; cricket-cookies and where it turned out that GriGri:s are great and saves lives. To Sina and André I would like to say that I have never had two such good friends. You are like brothers to me; lovely, caring and helpful at one moment, but bullying and annoying the next.

At last, thank you me. I did a good job! I might not be the fastest or the smartest but I have worked hard... and I do have the fluffiest hair and the most used socks! 



\section{Contents}

1 Introduction 1

1.1 Pose estimation in room-scaled environments . . . . . . . . . . 1

1.2 VPS: An application to metrology .................. 4

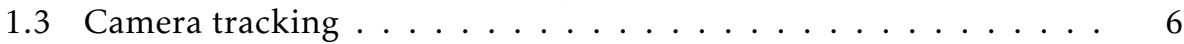

1.4 Publications and contributions . . . . . . . . . . . . 8

1.5 Thesis outline ....................... 11

\section{Background}

2 Filtering $\quad 15$

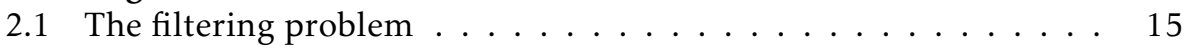

2.2 The Bayesian recursive filter . . . . . . . . . . . . . . . 16

2.2.1 Statistical system model . . . . . . . . . . . . 17

2.2.2 The estimate update recursion . . . . . . . . . . . 18

2.3 The Kalman filter for linear systems . . . . . . . . . . . . . . . . 19

2.3.1 Linear Gaussian state-space model . . . . . . . . . . . 20

2.3.2 The Kalman filter recursion . . . . . . . . . . . . . 20

2.4 Kalman filter approximations for nonlinear systems . . . . . . . 22

2.4.1 The extended Kalman filter . . . . . . . . . . . . 24

2.5 Simultaneous localization and mapping . . . . . . . . 26

3 The Cramér-Rao lower bound 29

3.1 CRLB for static systems . . . . . . . . . . . . . . . 29

3.1.1 Posterior CRLB for random parameters . . . . . . . . 30

3.1.2 Parametric CRLB for deterministic parameters . . . . . . . 31

3.2 CRLB for dynamic systems . . . . . . . . . . . . . . 32

3.2.1 Posterior CRLB for random state trajectory . . . . . . . . 32

3.2.2 Parametric CRLB for deterministic state trajectory . . . . . . 33

4 Motion modeling $\quad 37$

4.1 Coordinate frames . . . . . . . . . . . . . . . 37

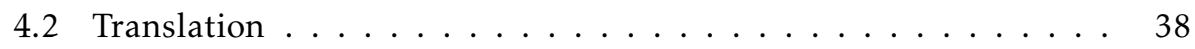




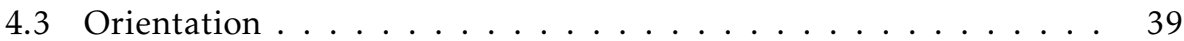

4.4 Combined translation and rotation . . . . . . . . . . . 40

4.4.1 Alternative 1: Model with additional states . . . . . . . . 41

4.4.2 Alternative 2: Model with direct inputs . . . . . . . . . 41

5 Sensor modeling 43

5.1 Sensor specific coordinate system . . . . . . . . . . . . . . . 43

5.2 Rotation matrices . . . . . . . . . . . . . . . . 44

5.3 Inertial measurement unit . . . . . . . . . . . . . . . . 44

5.3.1 Measurement model ............... . 45

5.3.2 Model errors . . . . . . . . . . . . . . . . 46

5.4 Optical camera . . . . . . . . . . . . . . . . . . . 47

5.4.1 Measurement model . . . . . . . . . . . . . 44 47

5.4 .2 Model errors . . . . . . . . . . . . . . . 50

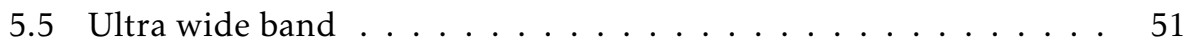

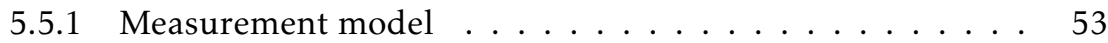

5.5 .2 Model errors . . . . . . . . . . . . . . . . 54

5.6 Detected known signals . . . . . . . . . . . . . . 56

5.6 .1 Measurement model . . . . . . . . . . . . . . 57

5.6 .2 Model errors . . . . . . . . . . . . . . 58

6 Concluding remarks $\quad 61$

$\begin{array}{ll}\text { Bibliography } & 65\end{array}$

\section{Publications}

A A High-Performance Tracking System based on Camera and IMU $\quad 79$ Bibliography .................... 100

B Pose Estimation Using Monocular Vision and IMU Aided with UWB 103 Bibliography .................... 127

C On Joint Range and Velocity Estimation in Detection and Ranging Sensors

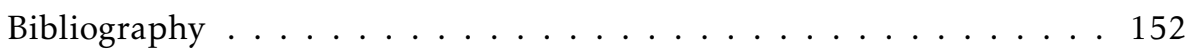




\section{1}

\section{Introduction}

Localization, navigation and tracking in large-scale, outdoor environments is a classical research area. Different global navigation satellite systems such as GPS nowadays allow for positioning with an accuracy of a couple of meters, or even a couple of centimeters with differential GPS, basically all over the world. Also, small-scaled tracking, such as tracking of computer desktop input devices like haptic mouses, is a mature field with many accurate commercial products. In the mid-scale we have localization, navigation and tracking in indoor multi-room environments, where the GPS do not reach. This is a research field that has gained a lot if interest lately with many emerging technologies. There is a missing piece in the research literature when it comes to tracking in room-scaled environments, on scales larger than a desk and smaller than a whole building. This thesis focuses on this missing piece. This chapter will take you through an introduction to pose tracking in room-scaled environments with its many interesting applications and provide a more detailed insight into the contributions and outline of this thesis.

\subsection{Pose estimation in room-scaled environments}

To be able to understand the problem addressed in this thesis, it is important to understand the meaning of the concept of "pose". The concept of "pose" is illustrated in Figure 1.1. An object existing in our three dimensional world can move with six degrees of freedom. It can change its position through translation and it can change its orientation through rotation. The combination of both position and orientation of an object is referred to as the pose of the object.

Tracking of pose is an enabling technology for many other areas. Virtual reality 


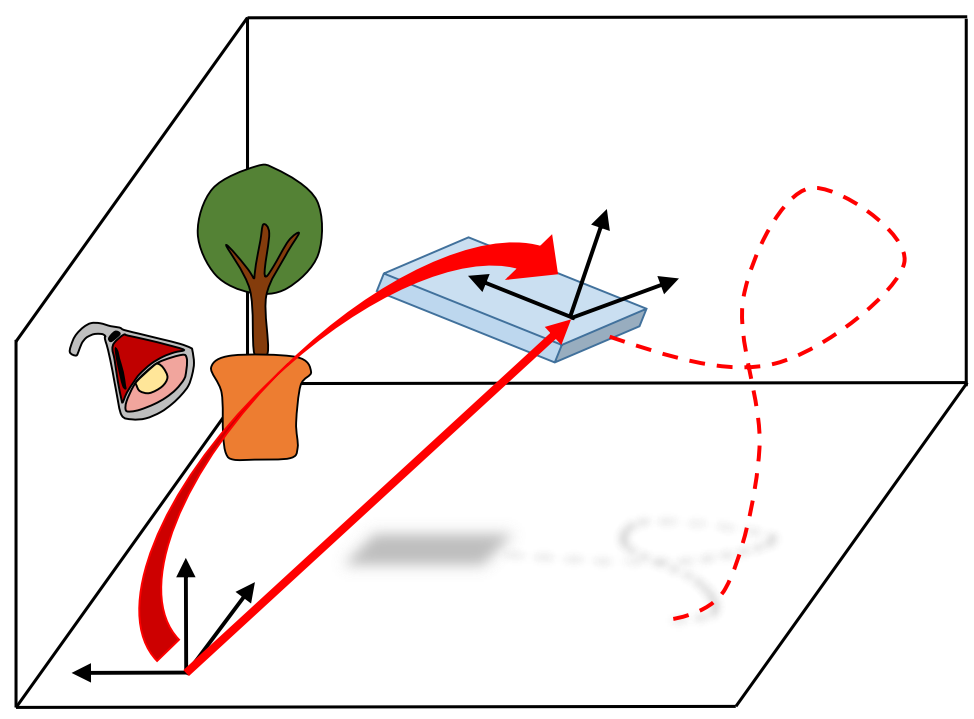

Figure 1.1: An illustration of the concept of pose as the position and orientation of an object relative to its surroundings.

(VR), computer simulations of a users presence in a made up world, or augmented reality (AR), real-time interaction with our physical world enhanced with computer generated sensory inputs, are classical examples. To create an experience that is realistic, it is crucial to track the movements of the user. High accuracy tracking is needed because the human brain is difficult to trick.

VR and AR have applications in many different areas, for example:

- entertainment such as gaming [53, 142], where for example Playstation VR, HTC Vive and Oculus Rift are big commercial names;

- support in everyday professions such as archaeology $[29,105]$, engineering, architecture and construction $[66,79,98,132]$, and medicin and rehabilitation $[61,65,92]$;

- education $[40,86]$; and

- military, and safety and rescue $[71,84]$.

These are just a few examples of the diversity and possibilities of VR and AR applications.

Tracking of pose is also useful for interacting with computer programs, machines and other tools $[36,55,89,91]$. Kinnect and the Wii controller are well known examples of commercial products used as input devices for game consols. Pose tracking can also be used for metrology, as the example in Section 1.2 will show, 
Table 1.1: Different applications and their pose accuracy requirements.

\begin{tabular}{lc}
\hline Application & Accuracy requirements \\
\hline Simple games & Approx. 1m, tens of ${ }^{\circ}$ \\
VR & A few dm, a few ${ }^{\circ}$ \\
AR & Approx. 1cm, approx. $1^{\circ}$ \\
Metrology & Sub-cm, sub- ${ }^{\circ}$ \\
\hline
\end{tabular}

Table 1.2: Examples of some commercial systems for tracking pose.

\begin{tabular}{lccc}
\hline Name & Intended use & Accuracy & Coverage \\
\hline $\begin{array}{l}\text { Ascension } \\
\text { trakSTAR }\end{array}$ & Reference system & $\begin{array}{c}\text { A couple of mm } \\
\text { Sub- }{ }^{\circ} \text { to }{ }^{\circ}\end{array}$ & Less than $2 \mathrm{~m}$ \\
Kinnect & $\begin{array}{c}\text { Motion sensing input } \\
\text { device to game console }\end{array}$ & $\begin{array}{c}\text { Approx. a dm } \\
\text { A couple of }{ }^{\circ}\end{array}$ & Approx $4 \mathrm{~m}$ \\
$\begin{array}{l}\text { Polhemus } \\
\text { SCOUT }\end{array}$ & Head tracking & $\begin{array}{c}\text { Sub-degree } \\
\text { Sub-mm }\end{array}$ & Approx. $2 \mathrm{~m}$ \\
Vicon & Reference system & $\begin{array}{c}\text { Sub- } \\
\text { Sum }\end{array}$ & A room \\
$\begin{array}{l}\text { Geomagic } \\
\text { Phantom }\end{array}$ & Input device & Sub- ${ }^{\circ}$ & Less than $1 \mathrm{~m}$ \\
\hline
\end{tabular}

and as ground truth reference systems [87].

The requirements on tracking accuracy varies between the applications as can be seen in Table 1.1. Note that the applications represented by each of the groups in the table puts different requirements on the precision needed. A game controller like Wii controller for example does not need to have as high accuracy as a controller for a surgical instrument. Examples of some existing commercial systems with their respective accuracies and intended use can be found in Table 1.2.

The basic principles of pose tracking can be divided into two groups:

- Self-sustaining methods: Sensors such as odometers and inertial measurement units (IMU:s) are self-sustaining sensors that can be used without the need for a structured environment with external infrastructure. This type of sensors measures relative motion such as acceleration, velocity, or angular velocity and can be used for dead reckoning. Pose estimates can hence be obtained from computing travelled distances or angles. Methods like this are in general good for estimation of fast motions over short time periods. Self-sustaining methods however tend to drift if used for longer periods of time due to the integration of measurement errors over time and the lack of usage of any absolute reference points in the environment.

Systems based on mechanical linkages do also exist. One end of the linkage is fixed and the other can be moved arbitrarily. Sensors in the linkage joints can measure the joint angles and the pose of the free end of the linkage can 
be computed. These types of systems can also be said to be self-sustaining but do not suffer from long term drift. They do however have a limited volume of operation and are too bulky for some applications.

- Methods using external infrastructure: Other sensors rely on the ability to find and identify absolute reference points, landmarks, in the environment. One example of such a sensor is vision cameras detecting bar-codes, markers, or object. A radio receiver (e.g. ultra wide band (UWB), WiFi, Bluetooth) detecting radio transmitters is another example, as is microphones which can be used to detect and localize sound sources. Sensors and methods like this do not suffer from the long term drift the self-sustaining methods suffer from. On the other hand, they require a structured environment with external infrastructure. This external infrastructure must be deployed, maintained and often also mapped before pose tracking can be performed. This makes these methods less user friendly and they are mostly used as stationary solutions that are not moved between different environments.

Which methods and sensors to use depends on the type on environment in which the object is moving. It is therefore important to also understand the concept of "room-scaled environment". A room-scaled environment is a limited space where an object can move freely in the order of meters in each direction, much like a room in a house. Mechanic tracking systems as described above can hence not be used due to their limited region of operation. Also, magnetic tracking such as [75] is excluded because of the same reason.

A room environment is also a semi-open space, with the prefix semi added because of the presence of smaller objects like furniture. This means that non-lineof-sight is not a big problem for radio or sound sensors but that occlusion can be a problem for vision sensors such as cameras. The presence of objects in the environment however opens up for vision systems that can take advantage of naturally appearing landmarks, such as objects or other environmental features that are easy to detect and recognize.

\subsection{VPS: An application to metrology}

One major funder of this study is the Virtual Photo Set (VPS) project [126]. The objective of this project is to measure the light field in an environment with a dedicated sensor and build models that can be used for scene reconstruction and efficient image synthesis. See Figure 1.2 for an illustration of the VPS application and the realism in the photo rendering.

Realistic environmental models have to be built as a part of the VPS scene reconstruction pipeline, as illustrated in Figure 1.2b. A camera is a complex sensor and the appearance of an image is affected by many different factors. An object can either emit light, reflect light or both. A camera captures both emitted and reflected light, as illustrated in Figure 1.3. This means that a good environmental model for scene reconstruction should contain both properties of light sources, 


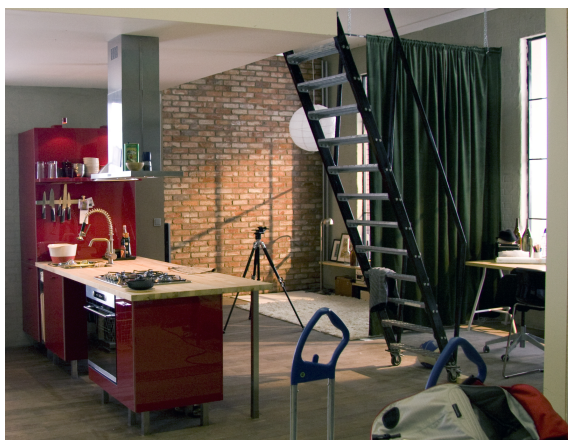

(a) The real photo studio environment.

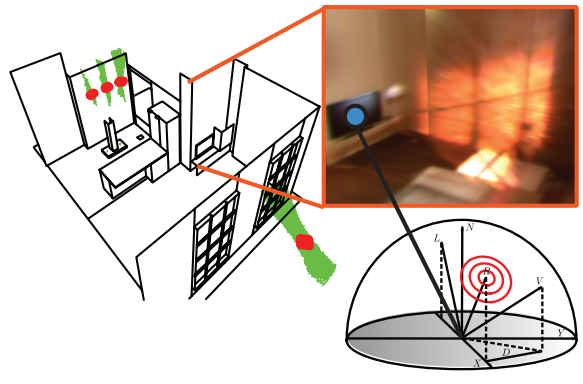

(b) The VPS model consist of a recovered geometric model of the scene that is textured with the photometric information from a HDR-video sequences and describes how the illumination varies between different locations in the scene.

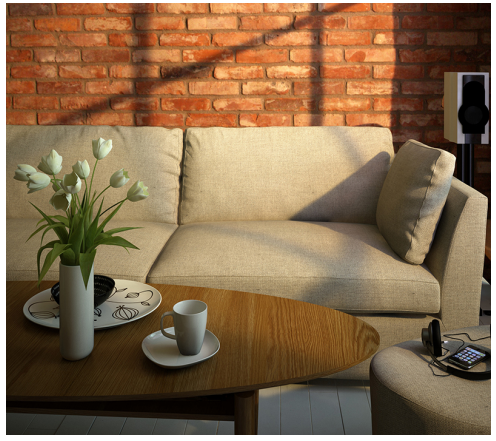

(d) A photo realistic rendering of the environment with the virtual furniture. (c) Virtual furniture can be placed in the recovered VPS model.

Figure 1.2: An example of the VPS application. 


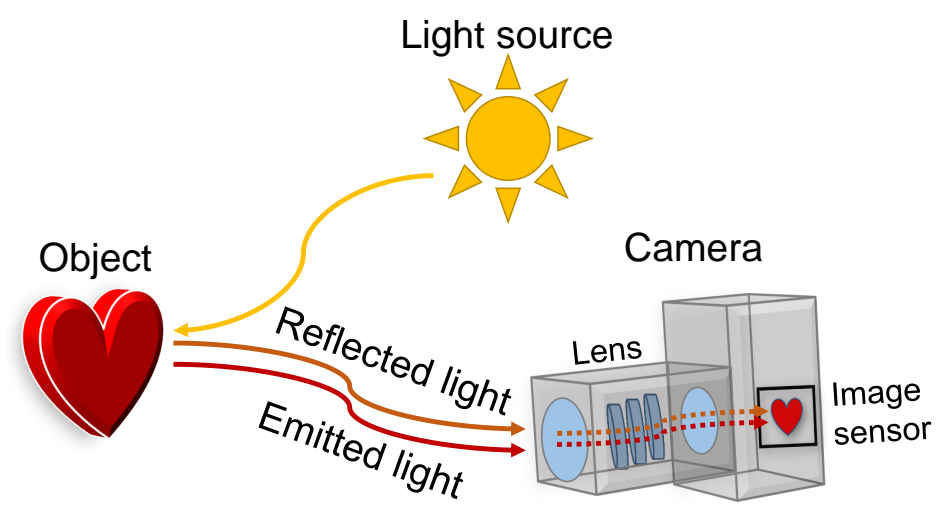

Figure 1.3: A three dimensional object can emit and/or reflect light. A camera captures this light on a two dimensional image sensor through a focusing lens.

as well as geometrical models of the environment and reflective properties of different surfaces.

Models of a camera can often be obtained through relatively simple calibration experiments that has do be performed only once per camera. Models of environmental factors such as light sources and reflective properties of objects are however a bit more difficult to obtain and has to be determined once per new photo studio. An important part of the VPS pipeline is to create these type of environmental models. For this, it is crucial to be able to track the pose of the camera that is used to collect the environmental data, as illustrated in Figure 1.4. Previously, a mechanical tracking system has been used. However, this is not optimal due to its limited volume of operation and because of the difficulty to move this system between different studios. A tracking system that is easy to use and move between different studios will improve the usefulness and the level of autonomy of the algorithms developed in the VPS project. Though, the required accuracy is high, approximately one millimeter for position and one degree for orientation.

\subsection{Camera tracking}

Because of the intended application to VPS and the big pool of possible applications for VR and AR, a camera will be used as a central part of the tracking methods developed in this thesis.

Early literature about camera tracking describes how known artificial markers can be used to compute the camera pose. ARToolKit [73] and its more recent improvement ARTag [30] are examples of software packages using these techniques that are still used today. Other examples are [117] describing a method for extract- 


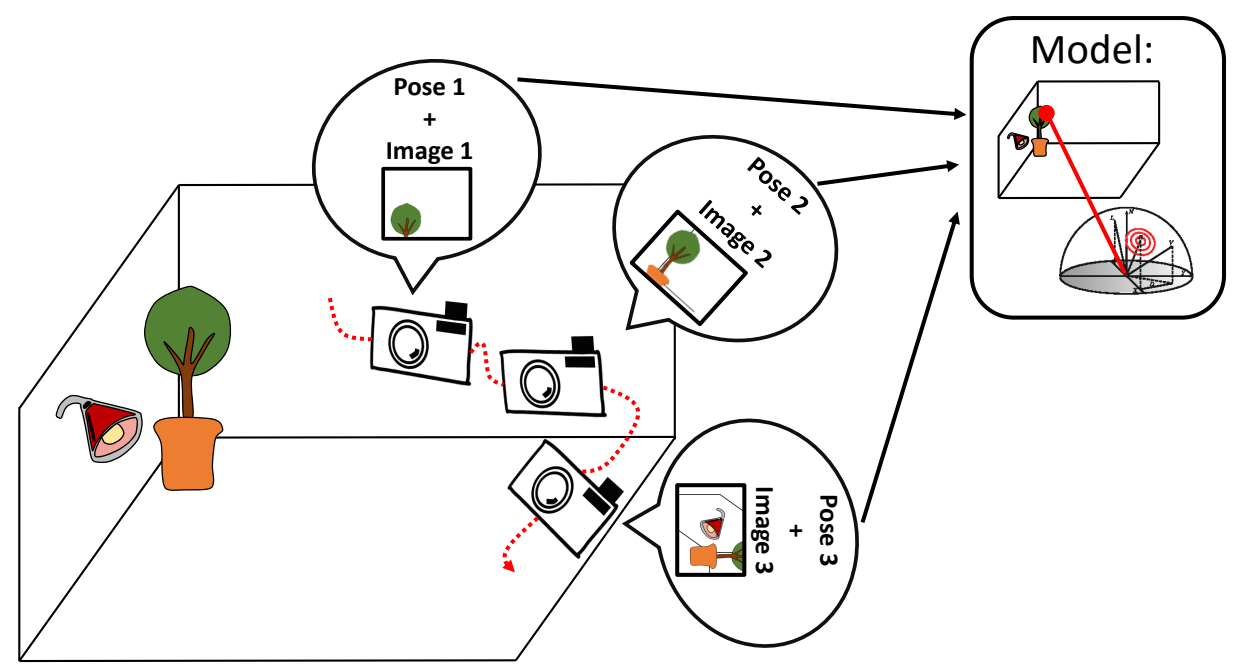

(a) Pose and photometric information are used to build the VPS models.
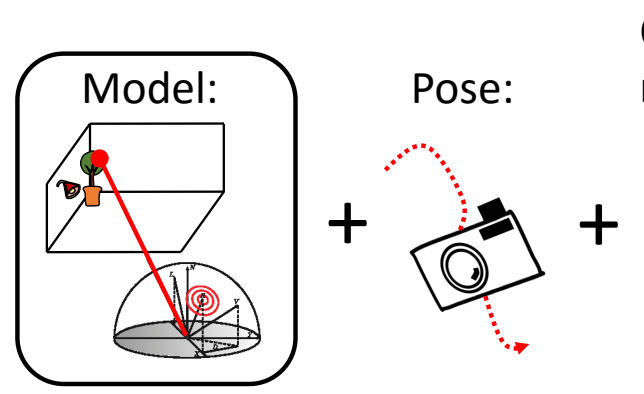

Object

(b) Once the VPS models are built, pose information, either simulated or obtained through live tracking of a real camera, can be used to render realistic images or videos containing virtual objects not present in the original scene.

Figure 1.4: An illustration of the importance of tracking of pose for the VPS project. 
ing and using corners of square markers for motion tracking and [106] focusing on increased robustness in the presence of outliers (incorrect marker detections).

Square shaped fiducial markers are common. Reference [138] compares some of the most used ones up until 2002, after which the research focus shifted somewhat and the use of fiducial markers decreased. However, other examples can also be found such as ring shaped [18] or circular shaped [130].

More recently, research on how naturally occurring landmarks, such as corners, edges, lines and textures, can be used for camera motion tracking has been a hot topic. Deploying and mapping fiducial markers can be difficult and time consuming and has to be performed in every new environment where a tracking system is to be used. Using naturally occuring landmarks opens up for more user friendly and mobile solutions. An early paper combining artificial and naturally occuring landmarks are [96], and more recent examples are [20,127, 140].

Model-based tracking has recently been the focus of the camera based tracking research. Simple features such as lines can be combined to describe more complex objects. With help of CAD models, 2D templates or other types of predefined models, these more complex objects can be identified and used as landmarks in a tracking solution $[24,107,110,137]$.

Research has also been conducted on hybrid systems where camera information is fused with information from other sensors. The inertial-camera combination has been the most popular hybrid combination over the years because of complementary properties of these sensors $[72,77,109,118,133]$. Other combinations has also been explored, for example fusion with GPS for outdoor tracking $[63,110]$.

\subsection{Publications and contributions}

The problem of camera pose tracking is, in this thesis, approached using sensor fusion. Complementary characteristics of sensors are exploited to get more accurate and more robust tracking with less need of support from external infrastructure. The three publications Paper A-C, written during 2013-2016, contain the following main contributions:

- Accurate modeling of inertial and camera measurements respectively, that can be used in applications where high accuracy is needed.

- Evaluation of the accuracy of an inertial-camera extended Kalman filter with pre-mapped fiducial markers in realistic, predefined user scenarios and conclusions about the requirements to achieve VPS accuracy.

- A novel approach to pose tracking where an inertial-camera system is aided with time-of-flight measurements from UWB radio signals. It is shown that this enables the use of naturally occurring visual landmarks rather than predefined fiducial markers. This drastically decreases the extent of the required external infrastructure without introducing error drift. 
- An informatics theoretical analysis of performance bounds and properties of an efficient range and velocity estimator used in a room-scaled environment resulting in conclusions about how these types of measurements should be used in a tracking filter for best performance. The theory is also applied to experimental acoustic data.

The contributions and background of each of the three publications respectively together with specifications of the contributions of the author of this thesis to the papers are clarified here.

\section{Paper A - A High-Performance Tracking System Based on Camera and IMU}

Edited version of the paper:

H. Nyqvist and F. Gustafsson. A high-performance tracking system based on camera and IMU. In 16th International Conference on Information Fusion (FUSION), pages 2065-2072, July 2013.

Summary: Paper A studies a system for indoor pose tracking with camera and IMU measurements using simulations. There exist many camera based tracking systems in literature and available commercially and a few of them are supported by an IMU. They are however based on the best-effort principle, where the performance varies depending on the situation and is known at best afterwards after evaluations of the tracking result. In contrast to this, Paper A starts with a specification of the system performance, and the design is based on an informatics theoretic approach, where specific user scenarios are defined. Precise models for the camera and IMU are derived for a fusion filter, and the theoretical Cramér-Rao lower bound and the extended Kalman filter performance is evaluated. The study in this paper focuses on examining the camera quality and the density and placement of virtual markers needed to get at least a one millimeter and one degree tracking accuracy, the accuracy needed in VPS.

Background and contributions: A mechanical tracking system has previously been used in VPS, but this approach suffered from a limited coverage of operation and was also difficult to move to new photo studios. Paper A is a first step to investigate if and how the tracking can be done in a simplified way with the same accuracy. The idea came from the second author of the paper, F. Gustafsson.

The code and the results presented in Paper A was created by the first author, the author of this thesis, with comments from F. Gustafsson. The author of this thesis also wrote Paper A with extensive comments and help from F. Gustafsson.

\section{Paper B - Pose Estimation Using Monocular Vision and Inertial Sensors Aided with Ultra Wide Band}

Edited version of the paper: 
H. E. Nyqvist, M. A. Skoglund, G. Hendeby, and F. Gustafsson. Pose estimation using monocular vision and inertial sensors aided with ultra wide band. In 2015 International Conference on Indoor Positioning and Indoor Navigation (IPIN), pages 1-10, October 2015. Runner up for the best paper award.

Summary: Paper B presents a method for global pose estimation using inertial sensors, monocular vision, and ultra wide band sensors. The complementary characteristics of these sensors are exploited to obtain improved global pose estimates, without requiring the introduction of any visible external infrastructure, such as fiducial markers. Naturally appearing visual landmarks are instead jointly estimated with the pose of the platform using a simultaneous localization and mapping framework while a small number of easy-to-hide ultra wide band beacons with known positions are used as support. The method is evaluated with data from a controlled indoor experiment with high precision ground truth.

Background and contributions: One requirement on the new tracking system in the VPS project is that is should be easy to move to new photo studios. One big disadvantage with the approach studied in Paper A is therefore that it requires knowledge about the placement of the fiducial markers used for the tracking. This, together with the fact that Paper A shows that a rather large number of markers have to be used in order for the required tracking accuracy to be reached, makes the approach infeasible. Paper B therefore studies if it is possible to make the need for external infrastructure smaller. To not lose the global observability and to reduce the drift in the estimates, the tracking system however needs some kind of global measurements. The choice fell on ultra wide band sensors since the authors found very little research done on camera-UWB hybrids.

The code used for Paper B paper was built on previous work done by $\mathrm{M}$. Skoglund but was rewritten and extended by the author of this thesis. The collection of the data used in Paper B was planned and executed by the author of this thesis together with G. Hendeby. All the results were created by the author of this thesis. The paper itself was written by the author of this thesis, M. Skoglund and G. Hendeby. F. Gustafsson gave extensive comments on the work throughout the whole process.

\section{Paper C - On Joint Range and Velocity Estimation in Detection and Ranging Sensors}

Edited version of the paper:

H. E. Nyqvist, G. Hendeby, and F. Gustafsson. On joint range and velocity estimation in detection and ranging sensors. In 19th International Conference on Information Fusion (FUSION), pages 16741681, July 2016.

Summary: In Paper C, range and radial velocity measurements obtained from 
reflected, known signals (comapare with for example radar and sonar) are explored. These measurements can be obtained from the round-trip time and Doppler shift of emitted signals. Estimation of the round-trip time and Doppler shift is usually done separately without considering the couplings between these two related quantities. In Paper $C$, the amplitude, time shift, and time scale of the returned signal is modeled in terms of range and velocity rather than in the more common time delay and Doppler shift parameters. This is because range and velocity are more natural parameters for tracking of moving objects. Then the Cramér-Rao lower bound for the joint range and velocity estimation problem is analyzed in order to get important information that can be used in a tracking filter to increase the tracking accuracy. The theory was also verified experimentally with data from sound pulses reflected in a wall in an indoor environment.

Background and contributions: Range and Doppler information from for example radars and sonars are used extensively for tracking in large scale outdoors environments. In smaller scale environments, like the once studied in this thesis, Doppler information is mostly neglected. For example radio (WiFi, UWB, Bluetooth), sound (audible or ultrasonic) or light (laser scanners) provides information about range but not velocity, even though it theoretically is possible to obtain. Paper $\mathrm{C}$ studies the possibility of obtaining both range and velocity measurement also in small scale environments. To be able to guarantee the high accuracy required in the VPS project we see it as crucial to obtain as much information as possible from the sensors we use.

The idea to Paper C came from F. Gustafsson. The data used in the paper was collected by the author of this thesis with support from G. Hendeby. The paper itself was written by the author of this thesis with extensive comments and support from G. Hendeby and F. Gustafsson.

\subsection{Thesis outline}

This thesis is divided into two parts, background material followed by a paper compilation.

Part one begins with a presentation of the theory for Bayesian estimation, its relation to linear and nonlinear Kalman filters and a short introduction to the simultaneous localization and mapping concept. After this follows a chapter about fundamental performance bounds of estimators. The last two chapters of the background presents dynamic models of the system studied in this thesis along with sensor models. The first part is ended with conclusions and discussions about the results and contributions of this thesis.

The second part contain slightly edited versions of the three papers that were briefly presented in Section 1.4 in chronological order. 

Part I

Background 



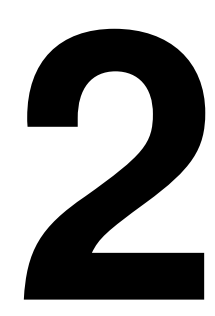

Filtering

A filter is an algorithm or a way to extract valuable information from measured signals while suppressing, for example, measurement errors or other misleading or irrelevant information. For the problem of tracking a moving object, a filter which exploits the change over time is necessary. This chapter will start with explaining the filtering problem and some of its difficulties. Then the Bayesian statistical framework for solving this problem will be explained. The perhaps most well known filter, the Kalman filter (KF) for linear Gaussian systems, and its relation to the Bayesian framework is explained next. This is followed by a description of how the KF framework can be extended to more difficult nonlinear problems with the extended Kalman filter (EKF) as a special case.

\subsection{The filtering problem}

The problem of filtering consists of estimating the states of a system as they are changing over time $[49,111]$. A system can be almost anything and in this thesis we consider a moving rigid body (a camera). What the states of the system represent of course depends on the type of system. For the case of a moving rigid body (camera) considered in this thesis, the states corresponds to pose and velocity of the body as modeled in Chapter 4.

A tracking filter should address the following important issues:

- As soon as new measurements or new information arrives it should be processed immediately and fast even when new information from sensors or other information sources arrive irregular.

- Sensors and other information sources are imperfect and provided measure- 
ments can not be completely trusted. Also, information might originate from different sources with different levels of reliability. Each individual information source might not measure the full information about the system states. If not, fusion of several sources is necessary. In case of outlier measurements, the obtained information can be contradictory and it is important to be able to detect which measurements to trust and which are the outliers.

- Individual measurements do not always give full information about the current state. Also, the obtained measurements might not give direct but only indirect knowledge about the states. For example, an IMU do not give information about pose directly, only indirectly through acceleration and angular velocity measurements.

- Important information about the state is lost if the information in past states is not properly accounted for. The states of the system are changing over time. However, states at different time instances are not uncorrelated with each other because of dynamic relationships and limitations of the system. Despite the dynamic behavior of the system it is not completely predictable. There might be errors in the model of the system or the system might be effected by disturbances which can not be anticipated or measured. Knowledge about system behavior has to be weighted against knowledge from sensors and other information sources.

In this thesis only discrete filters that operates on sampled signals represented in a digital form are considered. From now on a subscripts $k$ denote the sampling index of sampled signals. It does hence indicate the time $t_{k}$ at which the signal was sampled.

The filtering problem is illustrated in the block diagram in Figure 2.1. The same figure also introduces notation commonly used both in the control, signal processing and sensor informatics communities and which is also used throughout this thesis. The variable $x_{k}$ denotes the internal state of the system at time $t_{k}$ and the objective of a filter is to estimate these states. The output $\hat{x}_{k}$ from the filter corresponds to these state estimates. The variable $u_{k}$ is an input signal, which corresponds to known information about external impact on the system. $w_{k}$, on the other hand, is a disturbance signal and also affects the system behavior, similar to $u_{k}$, but corresponds to external impact that is not known, measurable, or possible to foresee. The sensor measurements are denoted by $y_{k}$ and the measurement errors are denoted by $e_{k}$. To simplify the notation we will throughout this thesis, without loss of generality, neglect the input signal $u_{k}$ and implicitly remember that this is a known model parameter.

\subsection{The Bayesian recursive filter}

Bayesian recursive filtering is a framework that addresses all of the issues with filtering mentioned above in Section 2.1 [113]. As soon as new measurements 


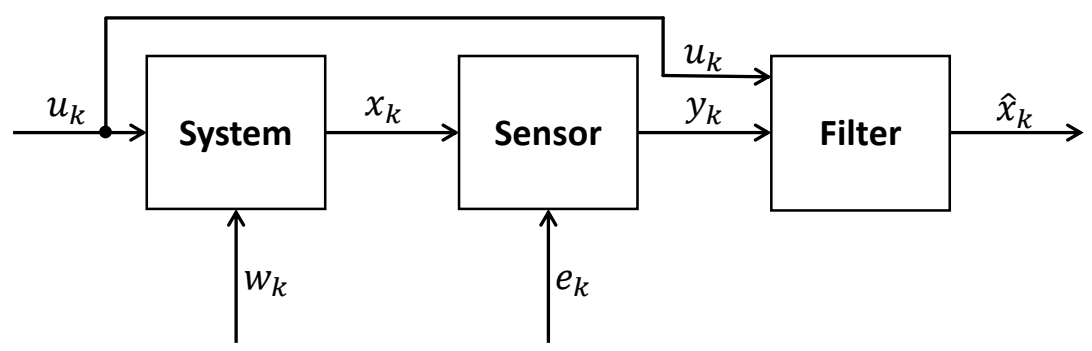

Figure 2.1: A block diagram illustrating the filtering problem.

are obtained, the state estimate can be updated through a recursive algorithm. In each recursion, the new measurements are used along with prior knowledge of the system dynamic behavior and previous state estimate. The uncertainties in the dynamic model, previous state estimates, and the measurements are handled through a statistical approach. Rather than representing states and measurements by single point estimates, they are represented with probability density functions (PDF:s) showing most likely values as well as uncertainties [35]. The statistical model is presented next followed by a description of the steps in the recursive state estimate update.

\subsubsection{Statistical system model}

Let $p(a)$ denote the PDF of the stochastic variable $a$ and $p(a \mid b)$ denote the PDF of the stochastic variable $a$ conditioned on the variable $b$. The system model used in the Bayesian recursive filter is then [67]

$$
\begin{aligned}
x_{k} & \sim p_{k}\left(x_{k} \mid x_{k-1}\right) \\
y_{k} & \sim p_{k}\left(y_{k} \mid x_{k}\right)
\end{aligned}
$$

with the prior

$$
x_{0} \sim p\left(x_{0}\right)
$$

for the initial state.

Equation (2.1a) above, the dynamic model, explains the state transition from time $t_{k-1}$ to time $t_{k}$. The PDF $p_{k}\left(x_{k} \mid x_{k-1}\right)$ should match uncertainties in the dynamic model caused by for example unpredictable disturbances. Equation (2.1b) above, the sensor model, connects the sensor measurements to the system states. The PDF $p_{k}\left(y_{k} \mid x_{k}\right)$ should match the uncertainties caused by measurement imperfections.

It should be noticed that the value of state $x_{k}$ depends only on the state $x_{k-1}$ one time instance in the past, not states further in the past, and not future states. Also, $x_{k}$ is only observed indirectly through observations $y_{k}$. This is illustrated in 


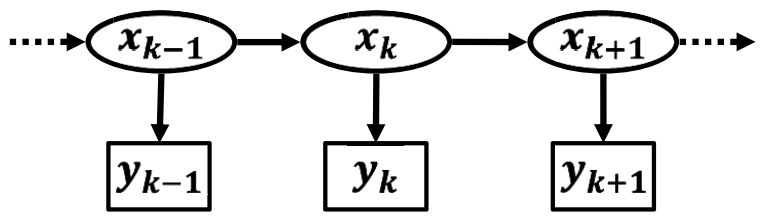

Figure 2.2: Illustration of a hidden Markov model. An arrow from a node to another shows how the variables effect each other.

Figure 2.2 and leads to [42]

$$
\begin{aligned}
p\left(x_{k} \mid x_{k-1}, x_{k-2}, \ldots, x_{0}\right) & =p\left(x_{k} \mid x_{k-1}\right) \\
p\left(y_{k} \mid x_{k}, x_{k-1}, \ldots, x_{0}\right) & =p\left(y_{k} \mid x_{k}\right) .
\end{aligned}
$$

A model like this is called a hidden Markov model.

\subsubsection{The estimate update recursion}

Here the principle of the Bayesian filtering will be explained and the presentation below is inspiered by $[67,113]$.

At time $t_{k}$, the true system state is $x_{k}$. At this time the filter has access to the measurements $\mathbb{Y}_{1: k}=\left\{y_{1}, y_{2}, \ldots, y_{k}\right\}$. Here $y_{k}$ is a new measurement obtained at time $k$ and $\mathbb{Y}_{1: k-1}=\left\{y_{1}, y_{2}, \ldots, y_{k-1}\right\}$ corresponds to all old measurements. The objective with the filter recursion is to compute the conditional PDF of the current state conditioned on the measurements up until the current time, $p\left(x_{k} \mid \mathbb{Y}_{1: k}\right)$.

The Markov property (2.2a) together with Bayes' equation

$$
p(a \mid b)=\frac{p(b \mid a) p(a)}{p(b)}
$$

and marginalisation

$$
p(a)=\int p(a, b) d b
$$

are keys to derive the Bayesian filter. With these components it is straightforward to see that $p\left(x_{k} \mid \mathbb{Y}_{1: k-1}\right)$ can be rewritten as

$$
p\left(x_{k} \mid \mathbb{Y}_{1: k-1}\right)=\int p_{k}\left(x_{k} \mid x_{k-1}\right) p\left(x_{k-1} \mid \mathbb{Y}_{1: k-1}\right) d x_{k-1}
$$

and that $p\left(x_{k} \mid \mathbb{Y}_{1: k}\right)$ can be rewritten as

$$
p\left(x_{k} \mid \mathbb{Y}_{1: k}\right)=\frac{p_{k}\left(y_{k} \mid x_{k}\right) p\left(x_{k} \mid \mathbb{Y}_{1: k-1}\right)}{\int p\left(y_{k} \mid x_{k}\right) p\left(x_{k} \mid \mathbb{Y}_{1: k-1}\right) d x_{k}},
$$

which correspond to the Bayesian recursive equations. The prior $p\left(x_{k-1} \mid \mathbb{Y}_{1: k-1}\right)$ (computed in the previous recursion) can hence be used to compute $p\left(x_{k} \mid \mathbb{Y}_{1: k-1}\right)$ in the time update(2.5a). Then, $p\left(x_{k} \mid \mathbb{Y}_{1: k-1}\right)$ can in turn be used in (2.5b) to com- 
pute the sought $p\left(x_{k} \mid \mathbb{Y}_{1: k}\right)$, which is used as a prior in the next recursion. The first filter recursions has to be initialized with the system model prior (2.1c).

As can be seen, the filter update is done in two steps. The first step is often referred to as the time update or the prediction step of the filter. The reason for this is that the new information $y_{k}$ is not used in this step. Instead this step corresponds to just a prediction of the state at the current time with help of only the prior from previous recursion and the dynamic model (2.1a). The second step of the recursion is often referred to as the measurement update because in this step the prediction from the time update is corrected based on the information carried by the new measurement and the sensor model (2.1b).

The filter provides estimates of the PDF of the filtered states. If instead point estimates of the state are required, it can be obtained for example as the conditional mean (minimum mean square error (MMSE) estimate),

$$
\hat{x}_{k \mid k}^{\mathrm{MMSE}}=\mathbb{E}_{x_{k}}\left\{x_{k} \mid \mathbb{Y}_{1: k}\right\}=\int x_{k} p\left(x_{k} \mid \mathbb{Y}_{1: k}\right) d x_{k},
$$

as the most likely value of the state (maximum a posteriori (MAP)),

$$
\hat{x}_{k \mid k}^{\mathrm{MAP}}=\underset{x_{x}}{\arg \max } p\left(x_{k} \mid \mathbb{Y}_{1: k}\right),
$$

or as other meaningful statistical measures.

\subsection{The Kalman filter for linear systems}

Already in 1960/61 Rudolf E. Kálmán published the papers [69, 70], which are often considered to be the origin of the famous Kalman filter (KF). However, for example Thorvald Nicolai Thiele did similar work already in 1880 [78] and Peter Swerling in 1958/59 [119, 120].

The KF is today widely used both in academia and by researchers as well as in industry. Although the first derivations of the KF was done with help of theories of optimal filtering from a frequentist's point of view, it can also be viewed as special case of the Bayesian recursive filter. The latter Bayesian view point is adopted in this thesis.

For the general statistical model (2.1) the Bayesian filter recursion equations (2.5) have no analytical solutions and must instead be computed numerically as done in for example the particle filter $[23,50]$. Though, for the special case when the statistical model (2.1) corresponds to a linear Gaussian state-space model, the recursion equations have analytical solutions which leads to the $\mathrm{KF}[8,60]$. The linear Gaussian state-space model will be described in the next section followed by the KF recursion. 


\subsubsection{Linear Gaussian state-space model}

The following constitutes a linear Gaussian state-space model [112]:

$$
\begin{aligned}
& x_{k}=A_{k} x_{k-1}+B_{k} w_{k-1} \\
& y_{k}=C_{k} x_{k}+D_{k} e_{k}
\end{aligned}
$$

with a Gaussian prior for the initial state,

$$
x_{0} \sim \mathcal{N}\left(x_{0} ; \hat{x}_{0}, P_{0}\right),
$$

and Gaussian disturbance and measurement noise,

$$
\begin{gathered}
w_{k} \sim p_{k}\left(w_{k}\right)=\mathcal{N}\left(w_{k} ; \mu_{k}^{w}, Q_{k}\right) \\
e_{k} \sim p_{k}\left(e_{k}\right)=\mathcal{N}\left(e_{k} ; \mu_{k}^{w}, R_{k}\right) .
\end{gathered}
$$

Note that mean and covariance are sufficient information to fully describe a Gaussian distribution [12].

A statistical model like (2.1), that can be used in a Bayesian recursive filter, can be obtained from this state-space model [112],

$$
\begin{aligned}
& x_{x} \sim p_{k}\left(x_{k} \mid x_{k-1}\right)=\mathcal{N}\left(x_{x} ; A_{k} x_{k-1}+B_{k} \mu_{k-1}^{w}, B_{k} Q_{k-1} B_{k}^{T}\right) \\
& y_{k} \sim p_{k}\left(y_{k} \mid x_{k}\right)=\mathcal{N}\left(y_{k} ; C_{k} x_{k}+D_{k} \mu_{k}^{e}, D_{k} R_{k} D_{k}^{T}\right) \\
& x_{0} \sim \mathcal{N}\left(x_{0} ; \hat{x}_{0}, P_{0}\right) .
\end{aligned}
$$

\subsubsection{The Kalman filter recursion}

The Bayesian recursive filter equations can be analytically computed for the case of the linear Gaussian state-space model described in Section 2.3.1. This analytical solution correspond to the KF solution to the filtering problem [60]. A key to understand this lies in realizing that the linear model (2.8) propagates Gaussian properties over time thanks to the theory of conjugate priors [12]. The predictions $p\left(x_{k} \mid \mathbb{Y}_{1: k-1}\right)$ and posteriors $p\left(x_{k} \mid \mathbb{Y}_{1: k}\right)$, computed in the Bayesian filter (2.5), are therefore Gaussian distributed for all times. They can consequently be fully parametrized by only two parameters, a mean and a covariance matrix. Let us define the means and the covariance matrices of the prediction and posterior PDF:s according to

$$
\begin{aligned}
p\left(x_{k} \mid \mathbb{Y}_{1: k-1}\right) & \triangleq \mathcal{N}\left(x_{k} ; \hat{x}_{k \mid k-1}, P_{k \mid k-1}\right) \\
p\left(x_{k} \mid \mathbb{Y}_{1: k}\right) & \triangleq \mathcal{N}\left(x_{k} ; \hat{x}_{k \mid k}, P_{k \mid k}\right) .
\end{aligned}
$$

The indexing $k_{1} \mid k_{2}$ is standard notation in KF literature and denotes "estimate at time $k_{1}$ given the measurements up until time $k_{2}$ ".

The equations (2.9a) and (2.10a) can then be used to compute the Bayesian time 
update (2.5a)

$$
\begin{aligned}
& \mathcal{N}\left(x_{k} ; \hat{x}_{k \mid k-1}, P_{k \mid k-1}\right)=p\left(x_{k} \mid \mathbb{Y}_{1: k-1}\right) \\
& =\int p_{k}\left(x_{k} \mid x_{k-1}\right) p\left(x_{k-1} \mid, \mathbb{Y}_{1: k-1}\right) d x_{k-1} \\
& =\int \mathcal{N}\left(x_{k} ; A_{k} x_{k-1}+B_{k} \mu_{k-1}^{w}, B_{k} Q_{k-1} B_{k}^{T}\right) \mathcal{N}\left(x_{k-1} ; \hat{x}_{k-1 \mid k-1}, P_{k-1 \mid k-1}\right) d x_{k-1} \\
& =\mathcal{N}\left(x_{k} ; A_{k} \hat{x}_{k-1 \mid k-1}+B_{k} \mu_{k-1}^{w}, A_{k} P_{k-1 \mid k-1} A_{k}^{T}+B_{k} Q_{k} B_{k}^{T}\right) .
\end{aligned}
$$

The measurement update equation $(2.5 \mathrm{~b})$ can similarly be rewritten as

$$
\begin{aligned}
\mathcal{N}\left(x_{k} ; \hat{x}_{k \mid k}, P_{k \mid k}\right) & =p\left(x_{k} \mid \mathbb{Y}_{1: k}\right) \\
& =\frac{p_{k}\left(y_{k} \mid x_{k}\right) p\left(x_{k} \mid \mathbb{Y}_{1: k-1}\right)}{\int p_{k}\left(y_{k} \mid x_{k}\right) p\left(x_{k} \mid \mathbb{Y}_{1: k-1}\right) d x_{k}} \\
& =\frac{\mathcal{N}\left(y_{k} ; C_{k} x_{k}+D_{k} \mu_{k}^{e}, D_{k} R_{k} D_{k}^{T}\right) \mathcal{N}\left(x_{k} ; \hat{x}_{k \mid k-1}, P_{k \mid k-1}\right)}{\int \mathcal{N}\left(y_{k} ; C_{k} x_{k}+D_{k} \mu_{k}^{e}, D_{k} R_{k} D_{k}^{T}\right) \mathcal{N}\left(x_{k} ; \hat{x}_{k \mid k-1}, P_{k \mid k-1}\right) d x_{k}} \\
& =\mathcal{N}\left(x_{k} ; \hat{x}_{k \mid k}+K_{k} \epsilon_{k},\left(I-K_{k} C_{k}\right) P_{k \mid k-1}\right),
\end{aligned}
$$

where the measurement residuals $\epsilon_{k}$ and the KF gain $K_{k}$ correspond to

$$
\begin{aligned}
\epsilon_{k} & =y_{k}-C_{k} \hat{x}_{k \mid k-1}-D_{k} \mu_{k}^{e} \\
\tilde{S}_{k} & =P_{k \mid k-1} C_{k}^{T} \\
S_{k} & =C_{k} \tilde{S}_{k}+D_{k} R_{k} D_{k}^{T} \\
K_{k} & =\tilde{S}_{k} S_{k}^{-1} .
\end{aligned}
$$

Comparison of the first and last equalities in (2.11a) and (2.11b) above respectively gives for the time update,

$$
\begin{aligned}
& \hat{x}_{k \mid k-1}=A_{k} \hat{x}_{k-1 \mid k-1}+B_{k} \mu_{k-1}^{w} \\
& P_{k \mid k-1}=A_{k} P_{k-1 \mid k-1} A_{k}^{T}+B_{k} Q_{k} B_{k}^{T},
\end{aligned}
$$

and for the measurement update,

$$
\begin{aligned}
& \hat{x}_{k \mid k}=\hat{x}_{k \mid k}+K_{k} \epsilon_{k} \\
& P_{k \mid k}=\left(I-K_{k} C_{k}\right) P_{k \mid k-1},
\end{aligned}
$$

which correspond to the famous KF recursion. The relationship between the Bayesian recursive filter and the KF should now hopefully be quite clear. With a linear Gaussian model, $p\left(x_{k} \mid \mathbb{Y}_{1: k-1}\right)$ and $p\left(x_{k} \mid \mathbb{Y}_{1: k}\right)$ are both Gaussian for all $k$. They are therefore fully describable by means and covariance matrices only, which are recursively computed in the KF.

The first filter recursion has to be initialized with the prior $(2.8 \mathrm{c})$ according to $\hat{x}_{0 \mid 0}=\hat{x}_{0}$ and $P_{0 \mid 0}=P_{0}$.

The first KF formulation by Rudolf E. Kálmán was not divided into the two sep- 
arate update steps. However, when doing the first digital KF implementation in the beginning of the sixties, Stanley F. Schmidt had to reformulate the filter and then identified the two update steps presented in this section $[46,88]$. The two-step formulation is very useful in practice. It for example allows for simple handling of nonuniform sampling, sensors and devices with different sampling time, and missing data since it is possible to do several time updates before a measurement update is performed and each measurement update may involve only a subset of all the available sensor. The same is of course true for the general two step Bayesian recursion (2.5). This property is used in Paper B where sensors with different and nonuniform sampling times are used.

Another thing, which makes the above formulation of the KF useful in practice, is that it provides a straightforward way of implementing measurement outlier detection. It is worth to notice that the variable $\tilde{S}_{k}$ in $(2.12 \mathrm{~b})$ actually corresponds to the cross-covariance matrix between the one step ahead predicted states and the predicted measurements and that $S_{k}$ in $(2.12 \mathrm{c})$ corresponds to the covariance matrix of the predicted measurements (this will be made clearer in Section 2.4). With access to the information in $S_{k}$, statistical tests that detects outlier measurements can be implemented. Outlier detectors using this technique is implemented in Paper B.

\subsection{Kalman filter approximations for nonlinear systems}

One disadvantage with the original formulation of the KF is that it is only applicable to systems that can be modeled as linear and Gaussian. Many systems are better described by nonlinear models

$$
\begin{aligned}
x_{k} & =f_{k}\left(x_{k-1}, w_{k-1}\right) \\
y_{k} & =h_{k}\left(x_{k}, e_{k}\right) \\
x_{0} & \sim p\left(x_{0}\right) \quad \text { with } \quad \hat{x}_{0} \triangleq \mathbb{E}_{x_{0}}\left\{x_{0}\right\} \text { and } P_{0} \triangleq \operatorname{Cov}_{x_{0}}\left\{x_{0}\right\} \\
w_{k} & \sim p\left(w_{k}\right) \quad \text { with } \quad \mu_{k}^{w} \triangleq \mathbb{E}_{w_{k}}\left\{w_{k}\right\} \text { and } Q_{k} \triangleq \operatorname{Cov}_{w_{k}}\left\{w_{k}\right\} \\
e_{k} & \sim p\left(e_{k}\right) \quad \text { with } \quad \mu_{k}^{e} \triangleq \mathbb{E}_{e_{k}}\left\{e_{k}\right\} \text { and } R_{k} \triangleq \operatorname{Cov}_{e_{k}}\left\{e_{k}\right\} .
\end{aligned}
$$

Due to the nonlinear state and measurement transformations, Gaussian properties of state and state estimates do not propagate over time as described in the KF section, Section 2.3. Approximate Kalman filters do nevertheless keep the Gaussian framework by approximating non-Gaussian distributions with Gaussian ones. How well these approximate KF work are therefore highly dependent on the severity of the nonlinearity and how well the distorted non-Gaussian distributions resembles their Gaussian approximations. Models involving multimodal PDF:s $p\left(x_{k} \mid x_{k-1}\right)$ and $p\left(y_{k} \mid x_{k}\right)$ are typically difficult to handle within the KF framework. For example the particle filter $[23,50]$, which is not limited to the unimodal Gaussian approximation, are likely to perform better for multimodal models. 
There are several suggested ways of how the Gaussian approximation can be done, for example with help of the first or second order Taylor expansions leading to the first or second order extended Kalman filter (EKF:s) [5, 47], the unscented transform leading to the unscented Kalman filter (UKF) [131] or the Monte Carlo transform leading to the Monte Carlo Kalman filter [51]. However, the algorithmic structure of all these filters is the same as explained in [52].

In the time update, the KF computes the mean, $\hat{x}_{k \mid k-1}$, and covariance, $P_{k \mid k-1}$ of $x_{k} \mid \mathbb{Y}_{1: k-1}$. The objective in an approximate KF filter is to compute this same properties. Noticing that the state transition equations (2.13) is a nonlinear transformation of $x_{k-1}$ and $w_{k-1}$, the help variables

$$
\begin{gathered}
\tilde{x}_{T U} \triangleq\left(\begin{array}{c}
x_{k-1} \\
w_{k-1}
\end{array}\right) \\
z_{T U}\left(\tilde{x}_{T U}\right) \triangleq x_{k}=f_{k}\left(x_{k-1}, w_{k-1}\right)
\end{gathered}
$$

are formed. The mean and covariance of $\tilde{x}_{T U}$ is given directly from computations of $\hat{x}_{k-1 \mid k-1}$ and $P_{k-1 \mid k-1}$ in the previous filter recursion and the disturbance model (2.13d). The Gaussian approximation of $\tilde{x}_{T U}$ is then

$$
\tilde{x}_{T U} \sim \mathcal{N}\left(\tilde{x}_{T U} ;\left(\begin{array}{c}
\hat{x}_{k-1 \mid k-1} \\
\mu_{k-1}^{w}
\end{array}\right),\left(\begin{array}{cc}
P_{k-1 \mid k-1} & 0 \\
0 & Q_{k-1}
\end{array}\right)\right) .
$$

Now any suitable approximation, for example any of the previously mentioned, can be used to compute approximate values of the mean and covariance of $z_{T U}$. Finally, the values of the sought $\hat{x}_{k \mid k-1}$ and $P_{k \mid k-1}$ can be obtained from this computed mean and covariance by noticing that $z_{T U}$, because of the definition in (2.14b), is approximately Gaussian according to

$$
z_{T U} \sim \mathcal{N}\left(z_{T U} ; \hat{x}_{k \mid k-1}, P_{k \mid k-1}\right) \text {. }
$$

The same approach is taken in the measurement update where the mean, $\hat{x}_{k \mid k}$, and covariance, $P_{k \mid k}$ of $x_{k} \mid \mathbb{Y}_{1: k}$ is sought. The help variables

$$
\begin{gathered}
\tilde{x}_{M U} \triangleq\left(\begin{array}{l}
x_{k} \\
e_{k}
\end{array}\right) \\
z_{M U}\left(\tilde{x}_{M U}\right) \triangleq\left(\begin{array}{l}
x_{k} \\
y_{k}
\end{array}\right)=\left(\begin{array}{c}
x_{k} \\
h_{k}\left(x_{k}, e_{x}\right)
\end{array}\right)
\end{gathered}
$$

is formed. The mean and covariance of $\tilde{x}_{M U}$ is given directly from computations of $\hat{x}_{k \mid k-1}$ and $P_{k \mid k-1}$ in the time update and the measurement model $(2.13 \mathrm{e})$. The Gaussian approximation of $\tilde{x}_{M U}$ is then

$$
\tilde{x}_{M U} \sim \mathcal{N}\left(\tilde{x}_{M U} ;\left(\begin{array}{c}
\hat{x}_{k \mid k-1} \\
\mu_{k}^{e}
\end{array}\right),\left(\begin{array}{cc}
P_{k \mid k-1} & 0 \\
0 & R_{k}
\end{array}\right)\right) .
$$

Again, any suitable approximation can be used to compute approximate values of the mean and covariance of $z_{M U}$. Now the properties $\hat{y}_{k \mid k-1}, \tilde{S}_{k}$ and $S_{k}$ can be 
defined and assigned values by noticing that $z_{M U}$, because of the definition in (2.17b), is approximately Gaussian according to

$$
z_{M U} \sim \mathcal{N}\left(z_{M U} ;\left(\begin{array}{l}
\hat{x}_{k \mid k-1} \\
\hat{y}_{k \mid k-1}
\end{array}\right),\left(\begin{array}{cc}
P_{k \mid k-1} & \tilde{S}_{k} \\
\tilde{S}_{k}^{T} & S_{k}
\end{array}\right)\right) .
$$

Note that $\tilde{S}_{k}$ corresponds to the cross-covariance between $x_{x}$ and and that $S_{k}$ corresponds to the covariance of $y_{k}$ just as in the linear KF in Section 2.3.

Finally, the values of the sought $\hat{x}_{k \mid k-1}$ and $P_{k \mid k-1}$ can be obtained from the KF equations $(2.12 \mathrm{~g})-(2.12 \mathrm{~h})$, repeated here

$$
\begin{aligned}
& \hat{x}_{k \mid k}=\hat{x}_{k \mid k}+K_{k} \epsilon_{k} \\
& P_{k \mid k}=\left(I-K_{k} C_{k}\right) P_{k \mid k-1},
\end{aligned}
$$

where $\epsilon_{k}$ and $K_{k}$ are computed as

$$
\begin{aligned}
\epsilon_{k} & =y_{k}-\hat{y}_{k \mid k-1} \\
K_{k} & =\tilde{S}_{k} S_{k}^{-1} .
\end{aligned}
$$

Similarly to the KF the first filter recursion is initialized with the prior $(2.13 \mathrm{c})$ according to $\hat{x}_{0 \mid 0}=\hat{x}_{0}$ and $P_{0 \mid 0}=P_{0}$.

It can be observed that this filter structure resembles the structure of the linear KF to a large extent. For the linear KF however, the $\hat{x}_{k \mid k-1}, P_{k \mid k-1}, \hat{x}_{k \mid k}$ and $P_{k \mid k}$ corresponds to the means and covariance matrices of the truly normal distributed PDF:s $p\left(x_{k} \mid \mathbb{Y}_{1: k-1}\right)$ and $p\left(x_{k} \mid \mathbb{Y}_{1: k}\right)$ while they for approximated nonlinear KF:s corresponds to the means and covariance matrices of their Gaussian approximations. Many nonlinear KF:s are equivalent to the linear KF for the linear Gaussian case, with the EKF and UKF as classical examples of this.

The EKF is perhaps the most well known approximate KF for nonlinear system models and will therefore be described in more detail in the next section.

\subsubsection{The extended Kalman filter}

The EKF is explained in for example [5, 47, 67]. The approximation approach taken in the (EKF) is to linearize the nonlinear state-space model around the current state estimate at each filter recursion. A linear model approximation is then obtained onto which the linear KF theory can be applied, see Section 2.3.

For the time update, a first order Taylor expansion of the help variable $z_{T U}$ around the mean of $\tilde{x}_{T U}$ gives

$$
\begin{aligned}
z_{T U} & \triangleq f_{k}\left(x_{k-1}, w_{k}\right) \\
& \approx f_{k}\left(\hat{x}_{k-1 \mid k-1}, \mu_{k-1}^{w}\right)+A_{k}\left(x_{k-1}-\hat{x}_{k-1 \mid k-1}\right)+B_{k} w_{k-1}
\end{aligned}
$$


where the matrices $A_{k}$ and $B_{k}$ are given by

$$
\begin{aligned}
& \left.A_{k} \triangleq \frac{\partial f_{k}(x, w)}{\partial x}\right|_{x=\hat{x}_{k-1 \mid k-1}, w=\mu_{k-1}^{w}} \\
& \left.B_{k} \triangleq \frac{\partial f_{k}(x, w)}{\partial w}\right|_{x=\hat{x}_{k-1 \mid k-1}, w=\mu_{k-1}^{w}} .
\end{aligned}
$$

This means that the linear theory presented in Section 2.3 can be applied and that the PDF of $z_{T U}$ can be approximated with

$$
z_{T U} \sim \mathcal{N}\left(z_{T U} ; f_{k}\left(\hat{x}_{k-1 \mid k-1}, \mu_{k-1}^{w}\right), A_{k} P_{k \mid k-1} A_{k}^{T}+B_{k} Q_{k-1} B_{k}^{T}\right) .
$$

Comparison with (2.16) and identification of the variables $\hat{x}_{k \mid k-1}$ and $P_{k \mid k-1}$ then gives

$$
\begin{aligned}
& \hat{x}_{k \mid k-1}=f_{k}\left(\hat{x}_{k-1 \mid k-1}, \mu_{k-1}^{w}\right) \\
& P_{k \mid k-1}=A_{k} P_{k \mid k-1} A_{k}^{T}+B_{k} Q_{k-1} B_{k}^{T}
\end{aligned}
$$

which corresponds to the time update of the EKF.

Similarly for the measurement update, a first order Taylor expansion of the help variable $z_{M U}$ around the mean of $\tilde{x}_{M U}$ gives

$$
z_{M U} \triangleq\left(\begin{array}{c}
x_{k} \\
h_{k}\left(x_{k}, e_{k}\right)
\end{array}\right) \approx\left(\begin{array}{c}
x_{k} \\
h_{k}\left(\hat{x}_{k \mid k-1}, \mu_{k}^{e}\right)+C_{k}\left(x_{k}-\hat{x}_{k \mid k-1}\right)+D_{k} e_{k}
\end{array}\right)
$$

where the matrices $C_{k}$ and $D_{k}$ are given by

$$
\begin{aligned}
& \left.C_{k} \triangleq \frac{\partial h_{k}(x, e)}{\partial x}\right|_{x=\hat{x}_{k-1 \mid k-1}, e=\mu_{k}^{e}} \\
& \left.D_{k} \triangleq \frac{\partial h_{k}(x, e)}{\partial e}\right|_{x=\hat{x}_{k-1 \mid k-1}, e=\mu_{k}^{e}} .
\end{aligned}
$$

The linear theory presented in Section 2.3 can again be applied and the PDF of $z_{M U}$ can be approximated as

$$
z_{M U} \sim \mathcal{N}\left(z_{M U} ;\left(\begin{array}{c}
\hat{x}_{k \mid k-1} \\
h_{k}\left(\hat{x}_{k \mid k-1}, \mu_{k}^{e}\right)
\end{array}\right),\left(\begin{array}{cc}
P_{k \mid k-1} & P_{k \mid k-1} C_{k}^{T} \\
C_{k} P_{k \mid k-1} & C_{k} P_{k \mid k-1} C_{k}^{T}+D_{k} R_{k} D_{k}^{T}
\end{array}\right)\right) .
$$

Comparison with (2.19) and identification of the variables $\hat{y}_{k \mid k-1}, \tilde{S}_{k}$ and $S_{k}$ then gives

$$
\begin{aligned}
\hat{y}_{k \mid k-1} & =h_{k}\left(\hat{x}_{k \mid k-1}, \mu_{k}^{e}\right) \\
\tilde{S}_{k} & =P_{k \mid k-1} C_{k}^{T} \\
S_{k} & =C_{k} P_{k \mid k-1} C_{k}^{T}+D_{k} R_{k} D_{k}^{T},
\end{aligned}
$$

which can be used in (2.20).

The EKF algorithm is used in both Paper A and B, where nonlinear but unimodal 


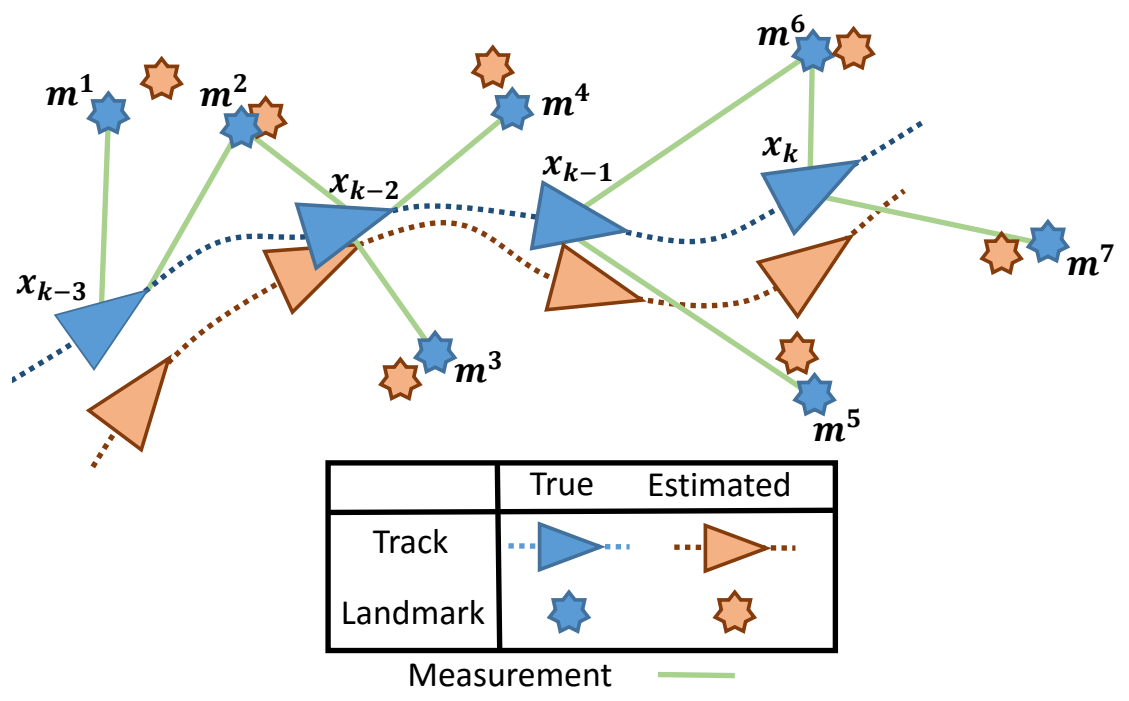

Figure 2.3: Illustration of the SLAM problem.

system and measurement models are used.

\subsection{Simultaneous localization and mapping}

As mentioned in the introduction, many principles for pose estimation rely on external infrastructure. For example camera tracking relies on finding and recognizing visual landmarks in the environment. External infrastructure needs maintenance, which may be time consuming or in other ways expensive. Simultaneous localization and mapping (SLAM) is a framework for building consistent maps of previously unknown environments and at the same time perform tracking within the map. It is by many seen as the key to making a system truly autonomous.

The measurements obtained during tracking depends on the external infrastructure. For the camera example, the appearance of a landmark in an image obviously depends not only of the pose of the camera itself, but also on the pose and appeararance of the landmark in the environment. Normally all parameters in the measurement model are assumed to be known, including the external infrastructure of landmarks. In the SLAM framework, the landmarks are instead seen as a part of the estimation problem as illustrated in Figure 2.3. The references $[7,26]$ gives a good overview both of the SLAM concept itself, the history of SLAM and existing solutions.

In [26] it is described how the SLAM problem can be stated in the Bayesian probabilistic framework, described in this chapter, by augmenting the state vector $x_{k}$ 
with the states of the unknown but static map of landmarks $m_{k}$

$$
x_{k}^{\mathrm{aug}}=\left(\begin{array}{c}
x_{k} \\
m_{k}
\end{array}\right)
$$

leading to the system state-space model

$$
\begin{aligned}
x_{k} & =f_{k}\left(x_{k-1}, w_{k-1}\right) \\
m_{k} & =m_{k-1} \\
y_{k} & =h_{k}\left(x_{k}, m_{k}, e_{k}\right) \\
x_{0} & \sim p\left(x_{0}\right) \\
m_{0} & \sim p\left(m_{0}\right) \\
w_{k} & \sim p\left(w_{k}\right) \\
e_{k} & \sim p\left(e_{k}\right) .
\end{aligned}
$$

The introduction of an unknown map creates some extra problems in addition to state estimation [7]:

- Since there is no prior information about the map, the number of landmarks is unknown. This means that the system state vector must be able to grow over times new landmarks are found. It also means that methods for initializing new landmarks into an the state vector must be available.

- Methods for associating a measurement to the correct landmark are also a crucial building block of a SLAM system. Incorrect associations may have devastating effects on the performance. Multi-hypotheses tracking [13] is a way of addressing the problem of uncertainty in the data association.

According to [26] the SLAM problem was recognized already in 1986. After a research breaktrough the first paper with a unifying structure and convergense results for the SLAM problem was published in 1996 [27]. Since then many different algorithms for solving the problem has been suggested. Some of the most important ones are the EKF-SLAM algorithm [48, 81], where the theory of EKF filtering is applied to the problem, and the FastSLAM algorithm [93, 94], where instead the Rao-Blackwellized particle filter [4] is applied.

The importance of solving the combined problem should be pointed out. Early work on the SLAM problem tended to focus on either mapping or localization. The breakthrough leading up the paper published in 1996 was the realization that the joint problem is convergent. Correlations between landmarks, as a cause of solving the joint problem, plays an important role and the bigger they grow the more accurate the solution becomes [26, 27]. 



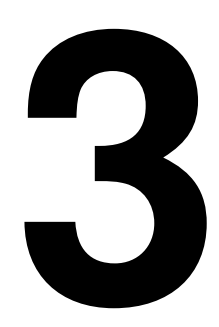

The Cramér-Rao lower bound

The nonlinear filtering problem often has to be solved through some kind of approximations, as seen in Chapter 2. This implies that the solution might be suboptimal. Comparing the performance of different filters can be difficult because it depends on the tracking scenario. For one scenario some filter may perform best, but for another scenario the same filter may get outperformed.

The Cramér-Rao lower bound (CRLB) is a theoretical lower bound on the covariance matrix of the estimation error obtainable by an estimator. Useful information about tracking performance can be obtained from comparisons with the CRLB. The closer to the CRLB the better the performance. If the performance of a filter is far from the CRLB, it might be worth investigating if better performance can be obtained by tuning the filter differently, or by switching to another filter type. An estimator that reaches the CRLB is said to be an efficient estimator.

There are different versions of the CRLB and it can be applied to both static and dynamic systems. Here the so called posterior and the parametric versions for static systems will be described first followed by an extension to dynamic systems.

\subsection{CRLB for static systems}

The theory of the CRLB for static systems is a well studied subject $[80,129]$. Let $\theta=\left[\begin{array}{llll}\theta^{(1)} & \theta^{(2)} & \ldots & \theta^{\left(n_{\theta}\right)}\end{array}\right]^{T}$ denote an unknown, $n_{\theta}$ dimensional, static variable that is to be estimated based on the measurement vector $\mathcal{Y}$. Under certain regularity conditions, the CRLB theory states that the covariance matrix of the estimate 
$\hat{\theta}(\mathcal{Y})$ are bounded by the CRLB according to

$$
\operatorname{Cov}_{\mathcal{Y}}\{\hat{\theta}(\mathcal{Y})\} \triangleq \mathbb{E}_{\mathcal{Y}}\left\{\left(\hat{\theta}(\mathcal{Y})-\mathbb{E}_{\mathcal{Y}}\{\hat{\theta}(\mathcal{Y})\}\right)\left(\hat{\theta}(\mathcal{Y})-\mathbb{E}_{\mathcal{Y}}\{\hat{\theta}(\mathcal{Y})\}\right)^{T}\right\} \geq P^{\text {CRLB }}
$$

The symbol $\geq$ means that $\operatorname{Cov} \mathcal{Y}\{\hat{\theta}(\mathcal{Y})\}-P_{\text {CRLB }}$ is positive definite.

$P_{\text {CRLB }}$ corresponds to the inverse of the $n_{\theta} \times n_{\theta}$ information matrix $J$,

$$
P^{\mathrm{CRLB}}=J^{-1} \text {. }
$$

The information matrix $J$ can be computed from statistical models of the parameter $\theta$ and measurement vector $\mathcal{Y}(\theta)$. Given knowledge about the parameters, the measurements $\mathcal{Y}$ are given by $p(\mathcal{Y} \mid \theta)$. The parameter vector $\theta$ can however be seen as either deterministic, or random in which case the true parameters are seen as a realisation of $p(\theta)$. Depending on the view taken the computations of $J$ and the regularity conditions under which (3.1) holds differs.

\subsubsection{Posterior CRLB for random parameters}

For the case of a random parameter vector $\theta$ the joint probability distribution of the measurements and the parameters can be formed as

$$
p(\mathcal{Y}, \theta)=p(\mathcal{Y} \mid \theta) p(\theta)
$$

The CRLB (3.1) holds under the conditions that [129]

1. $\frac{\partial p(\mathcal{Y}, \theta)}{\partial \theta^{(i)}}$ exist and is absolutely integrable with respect to $\mathcal{Y}$ and $\theta$ for all $i=1,2, \ldots, n_{\theta}$.

2. $\frac{\partial^{2} p(\mathcal{Y}, \theta)}{\partial \theta^{(i)} \partial \theta^{(j)}}$ exist and is absolutely integrable with respect to $\mathcal{Y}$ and $\theta$ for all $i, j=1,2, \ldots, n_{\theta}$.

3. The following

$$
\lim _{\theta^{(i)} \rightarrow \pm \infty} b(\theta) p(\theta)=0 \quad \text { for } i=1,2, \ldots, n_{\theta}
$$

holds for the conditional expectation of the estimation error (i.e. , the estimation bias) $b(\theta)$ given by

$$
b(\theta)=\mathbb{E}_{\mathcal{Y}}\{\hat{\theta}(\mathcal{Y})-\theta\} .
$$

Then the information matrix $J$ can be computed according to [80,129]

$$
\left[J^{\text {post }}\right]_{i, j}=\mathbb{E}_{\mathcal{Y}, \theta}\left\{\frac{\partial \ln p(\mathcal{Y}, \theta)}{\partial \theta^{(i)}} \frac{\partial \ln p(\mathcal{Y}, \theta)}{\partial \theta^{(j)}}\right\}=-\mathbb{E}_{\mathcal{Y}, \theta}\left\{\frac{\partial^{2} \ln p(\mathcal{Y}, \theta)}{\partial \theta^{(i)} \partial \theta^{(j)}}\right\},
$$

where $[\cdot]_{i, j}$ denotes the element on the $i$ :th row and in the $j$ :th column of a matrix. This information matrix is often referred to as the Bayesian information matrix (BIM). The BIM matrix was derived by [128] and independently by [115]. The second equality in (3.5) is not obvious but a proof can be found in [58]. 


\subsubsection{Parametric CRLB for deterministic parameters}

For a deterministic parameter vector $\theta$ the conditions under which (3.1) holds are slightly different [129]

1. $\frac{\partial \ln p(\mathcal{Y} \mid \theta)}{\partial \theta^{(i)}}$ exist and is absolutely integrable with respect to $\mathcal{Y}$ and $\theta$ for all $i=1,2, \ldots, n_{\theta}$.

2. $\frac{\partial^{2} \ln p(\mathcal{Y} \mid \theta)}{\partial \theta^{(i)} \partial \theta^{(j)}}$ exist and is absolutely integrable with respect to $\mathcal{Y}$ and $\theta$ for all $i, j=1,2, \ldots, n_{\theta}$.

3. The estimator is unbiased, hence $b(\theta)$ from (3.4) equals 0 for all $\theta$ and $\mathbb{E}_{\mathcal{Y}}\{\hat{\theta}(\mathcal{Y})\}=\theta^{*}$. Here $\theta^{*}$ denotes the true parameter values.

The information matrix $J$ is for the deterministic case computed according to $[80,129]$

$$
\left[J^{\text {par }}\right]_{i, j}=\left.\mathbb{E}_{\mathcal{Y}}\left\{\frac{\partial \ln p(\mathcal{Y} \mid \theta)}{\partial \theta^{(i)}} \frac{\partial \ln p(\mathcal{Y} \mid \theta)}{\partial \theta^{(j)}}\right\}\right|_{\theta=\theta^{*}}=-\left.\mathbb{E}_{\mathcal{Y}}\left\{\frac{\partial^{2} \ln p(\mathcal{Y} \mid \theta)}{\partial \theta^{(i)} \partial \theta^{(j)}}\right\}\right|_{\theta=\theta^{*}} .
$$

This information matrix is often referred to as the Fischer information matrix (FIM) $[32,33]$. The second equality in $(3.6)$ is not obvious but a proof can be found in [58].

Note that, instead of taking the expected value of all possible parameters $\theta$, as done in (3.5), here the expression is evaluated in the true parameter values $\theta^{*}$. This means that to be able to compute the parametric CRLB, ground truth must be available. It is a bit contradictory to both estimate the parameters and at the same time see it as known, but for some cases it is not. An example of when it is not contradictory is when estimation performance is evaluated compared to known ground truth in simulations or real experiments.

The connection between the BIM expression (3.5) and the FIM expression (3.6) should be better clarified. Note that the BIM expression, with help of (3.2), can be rewritten as $[124,129]$

$$
\begin{aligned}
{\left[J^{\text {post }}\right]_{i, j} } & =-\mathbb{E}_{\mathcal{Y}, \theta}\left\{\frac{\partial^{2} \ln p(\mathcal{Y} \mid \theta)}{\partial \theta^{(i)} \partial \theta^{(j)}}\right\}-\mathbb{E}_{\theta}\left\{\frac{\partial^{2} \ln p(\theta)}{\partial \theta^{(i)} \partial \theta^{(j)}}\right\} \\
& =-\mathbb{E}_{\theta}\left\{\left[J^{\text {par }}\right]_{i, j}\right\}-\mathbb{E}_{\theta}\left\{\frac{\partial^{2} \ln p(\theta)}{\partial \theta^{(i)} \partial \theta^{(j)}}\right\}
\end{aligned}
$$

For the deterministic case we have that $\theta=\theta^{*}$ and that $p(\theta)$ therefore is a constant. This means that the second term in (3.7) vanishes after computing the derivative and that the first term in (3.7) is evaluated in the single possible value of $\theta$ rather than averaged over several possible values, which results in the FIM expression.

Since the posterior CRLB is obtained by taking the expected value of all possible parameter values $\theta$, the parametric CRLB for one specific parameter value $\theta^{*}$ might actually be lower. Similarly, an estimator evaluated solely for one single $\theta^{*}$ 
(with different measurement noise realisations) might never reach the posterior CRLB. Hence, the parametric CRLB can in fact give a more accurate view of what can be expected from an estimator when the ground truth is known.

The CRLB cannot only be used as a benchmark, but also as a tool for analyzing or designing systems $[57,59,95]$. In Paper C, the parametric CRLB is used as an analyzing tool. The paper explores how reflected sound signals from an indoor environment should be used in a filtering context.

\subsection{CRLB for dynamic systems}

For the filtering case the parameters to estimate at each recursion correspond to the states of the system at time $k, \theta=x_{k}$. The available measurements at time $k$ are $\mathcal{Y}=\mathcal{Y}_{1: k}=\left\{y_{1}, y_{2}, \ldots, y_{k}\right\}$. All these measurements, both past $\mathcal{Y}_{1: k-1}$ and current $y_{k}$, contain information about the current state $x_{k}$. this is because previously collected measurements are linked to the current state through the dynamic behavior of the system as discussed in Chapter 2.

The system models used for filtering, presented in Chapter 2, actually give random models of state trajectories. The posterior CRLB can provide a lower bound that is averaged over all possible state trajectories. In contrast to this, the parametric CRLB framework sees the true state trajectory as known and deterministic. The papers $[74,123]$ gives a good insight in early work done on estimation bounds for nonlinear filtering, including parametric CRLB, while $[10,124]$ presents more recent work with focus on posterior CRLB.

How the parametric CRLB framework can be used for estimators of filtering kind will now be explained in more detail.

\subsubsection{Posterior CRLB for random state trajectory}

For the case of a dynamic system and a random state trajectory the BIM $J^{\text {post }}$ are given by

$$
\left[J^{\text {post }}\right]_{i, j}=-\mathbb{E}_{\mathbb{Y}_{1: k}, x_{k}}\left\{\frac{\partial^{2} \ln p\left(\mathbb{Y}_{1: k}, x_{k}\right)}{\partial x_{k}^{(i)} \partial x_{k}^{(j)}}\right\} .
$$

The references $[10,124,129]$ derives and presents a recursive expressions for computing this BIM,

$$
J_{k \mid k}^{\text {post }}=Q_{k-1}^{-1}+R_{k}^{-1}-S_{k-1}^{T}\left(J_{k-1 \mid k-1}^{\text {post }}+V_{k-1}\right)^{-1} S_{k-1},
$$


where the elements on the $i$ :th row and the $j$ :th column of the matrices $V_{k-1}, S_{k-1}$, $R_{k-1}^{-1}$ and $Q_{k-1}^{-1}$ respectively are computed as

$$
\begin{aligned}
& {\left[V_{k-1}\right]_{i, j}=-\mathbb{E}_{x_{k-1}, w_{k-1}}\left\{\frac{\partial^{2} \ln p\left(x_{k} \mid x_{k-1}\right)}{\partial x_{k-1}^{(i)} \partial x_{k-1}^{(j)}}\right\}} \\
& {\left[S_{k-1}\right]_{i, j}=-\mathbb{E}_{x_{k-1}, w_{k-1}}\left\{\frac{\partial^{2} \ln p\left(x_{k} \mid x_{k-1}\right)}{\partial x_{k-1}^{(i)} \partial x_{k}^{(j)}}\right\}} \\
& {\left[R_{k}^{-1}\right]_{i, j}=-\mathbb{E}_{x_{k}, y_{k}}\left\{\frac{\partial^{2} \ln p\left(y_{k} \mid x_{k}\right)}{\partial x_{k}^{(i)} \partial x_{k}^{(j)}}\right\}} \\
& {\left[Q_{k-1}^{-1}\right]_{i, j}=-\mathbb{E}_{x_{k-1}, w_{k-1}}\left\{\frac{\partial^{2} \ln p\left(x_{k} \mid x_{k-1}\right)}{\partial x_{k}^{(i)} \partial x_{k-1}^{(j)}}\right\} .}
\end{aligned}
$$

Recall also the regularity conditions presented in Section 3.1.1.

The recursion (3.9) holds under the assumption that the involved differentiations and expectations exist ${ }^{1}$. More specifically, $p\left(x_{k} \mid x_{k-1}\right)$ must be well defined for all times $k$, meaning that it must be twice differentiable with respect to both $x_{k}$ and $x_{k-1}$ [10]. A necessary condition for this to hold is that the disturbance vector $w_{k}$ has the same dimensionality as the state vector $x_{k}$. This is a very restrictive assumption because it is common that so is not the case.

The recursion (3.9) is initialized with help of the given prior distribution $p\left(x_{0}\right)$ according to $J_{0 \mid 0}^{\text {post }}=\left(\operatorname{Cov}_{x_{0}}\left\{x_{0}\right\}\right)^{-1}$.

\subsubsection{Parametric CRLB for deterministic state trajectory}

For the parametric CRLB, the state trajectory is assumed to be known and deterministic. A way of incorporating the trajectory knowledge into the parametric CRLB framework is to introduce fictitious, exact measurements $\mathcal{Z}_{1: k}=\left[\begin{array}{llll}z_{1}^{T} & z_{2}^{T} & \ldots & z_{k}^{T}\end{array}\right]^{T}$ of the true trajectory as discussed in for example [10, 37]. The fictitious measurements contain enough information to compute the true trajectory. As an example, the true initial state and the true system disturbance noise can be used as fictitious measurements.

The available measurements do then no longer consist of only $\mathcal{Y}_{1: k}$ but also the fictitious measurements $\mathcal{Z}_{1: k}$ that can constrain the evaluation of the parametric CRLB to the true trajectory. The elements of the FIM matrix $J^{\text {par }}$, for the case of a dynamic model with a deterministic state trajectory, is then hence computed according to

$$
\left[J^{\mathrm{par}}\right]_{i, j}=-\mathbb{E}_{\mathcal{Y}_{1: k}, \mathcal{Z}_{1: k}}\left\{\frac{\partial^{2} \ln p\left(\mathcal{Y}_{1: k}, \mathcal{Z}_{1: k} \mid x_{k}\right)}{\partial x_{k}^{(i)} \partial x_{k}^{(j)}}\right\}
$$

\footnotetext{
${ }^{1}$ Note that there is no need for $R_{k}$ and $Q_{k}$ to exist as long as $R_{k}^{-1}$ and $Q_{k}^{-1}$ do
} 
It has been shown for example by [10] that, for the general nonlinear state-space model (2.13), the filtering parametric CRLB matrix (inverse of FIM matrix) can be computed recursively according to $[10,58]$

$$
\begin{aligned}
& P_{k \mid k-1}^{\mathrm{CRLB}}=A_{k} P_{k-1 \mid k-1}^{\mathrm{CRLB}} A_{k}^{T}+B_{k} Q_{k-1} B_{k}^{T} \\
& P_{k \mid k}^{\mathrm{CRLB}}=P_{k \mid k-1}^{\mathrm{CRLB}}-\left(P_{k \mid k-1}^{\mathrm{CRLB}}\right)^{T} C_{k}^{T}\left(C_{k} P_{k \mid k-1}^{\mathrm{CRLB}} C_{k}^{T}+R_{k}\right)^{-1} C_{k}\left(P_{k \mid k-1}^{\mathrm{CRLB}}\right)^{T},
\end{aligned}
$$

where the matrices above are defined as

$$
\begin{aligned}
& A_{k}\left.\triangleq \frac{\partial f_{k}\left(x_{k-1}, w_{k-1}\right)}{\partial x_{k-1}}\right|_{x_{k-1}=x_{k-1}^{*}, w_{k-1}=w_{k-1}^{*}} \\
&\left.B_{k} \triangleq \frac{\partial f_{k}\left(x_{k-1}, w_{k-1}\right)}{\partial w_{k-1}}\right|_{x_{k-1}=x_{k-1}^{*}, w_{k-1}=w_{k-1}^{*}} \\
& {\left[Q_{k-1}^{-1}\right]_{i, j} \triangleq-\left.\mathbb{E}_{x_{k-1}}\left\{\frac{\partial^{2} p_{k}\left(x_{k-1}, w_{k-1}\right)}{\partial w_{k-1} \partial w_{k-1}}\right\}\right|_{w_{k-1}=w_{k-1}^{*}} } \\
& {\left[C_{k}^{T} R_{k}^{-1} C_{k}\right]_{i, j} \triangleq-\left.\mathbb{E}_{y_{k}}\left\{\frac{\partial^{2} p_{k}\left(y_{k} \mid x_{k}\right)}{\partial x_{k} \partial x_{k}}\right\}\right|_{x_{k}=x_{k}^{*}} }
\end{aligned}
$$

and where the factorization between $C_{k}$ and $R_{k}$ is done such that $R_{k}$ is positive definite. Recall aslo the regularity conditions presented in Section 3.1.2.

The recursion (3.11) hold under the assumption that all mentioned gradients, expectations and inverses exist. The recursion is initialized with help of the given prior distribution $p\left(x_{0}\right)$ according to $P_{0 \mid 0}^{\mathrm{CRLB}}=\operatorname{Cov}_{x_{0}}\left\{x_{0}\right\}$.

In this thesis the parametric version of the CRLB is preferred over the posterior when computing filtering performance bounds mainly because of three reasons.

1. For a single known trajectory belonging to the set of all possible trajectories the parametric CRLB gives a more fair view of the reality experienced in real data experiments as discussed in Section 3.1.2.

2. The parametric CRLB is often more easily computed than the posterior.

3. The parametric CRLB recursion (3.11) is not restricted to systems where the disturbance and state vectors have the same dimensionality, as the posterior CRLB recursion (3.9) is.

\section{Special case: Additive measurement noise}

A very commonly appearing model is to have additive measurement noise

$$
y_{k}=h\left(x_{k}\right)+e_{k}
$$

and Gaussian prior, disturbance and measurement noise models $p\left(x_{0}\right), p_{k}\left(w_{k}\right)$ and $p_{k}\left(e_{k}\right)$ (note that the disturbance noise $w_{k}$ can enter nonlinearly in the dynamic model).

For this special case the expressions (3.11e) and (3.11f) can be computed analytically [10]. Then $Q_{k-1}$ and $R_{k}$ in (3.11) corresponds to the covariance matrices 
of the Gaussian disturbance and measurement noise respectively and can be obtained directly from the system model. Also, the matrix $C_{k}$ can, similar to the matrices $A_{k}$ and $B_{k}$ in $(3.11 \mathrm{c})-(3.11 \mathrm{~d})$, be obtained from gradient operations on the system model,

$$
\left.C_{k} \triangleq \frac{\partial h_{k}\left(x_{k}\right)}{\partial x_{k}}\right|_{x_{k}=x_{k-1}^{*}} .
$$

The resemblance between this CRLB recursion and the covariance recursion used in the EKF should be noticed. The only difference is that in the EKF matrices $A_{k}$, $B_{k}$ and $C_{k}$ are computed through evaluation of the model gradients around the current state estimate instead of the around the true state since the filter does not have knowledge about the ground truth. Hence, if an EKF can provide state estimates close to the ground truth then the filter will likely be close to efficient. There are however no guarantees of the efficiency of the EKF.

This special case of the parametric CRLB was applied in Paper A where different user scenarios and the performance of an interial-camera EKF was evaluated.

\section{Special case: Linear Gaussian model}

For the special case of the linear Gaussian model (2.8), all the matrices $A_{k}, B_{k}, C_{k}$, $Q_{k-1}$ and $R_{k}$ in (3.11) can be obtained directly from the system model (2.8) and no gradient computations are needed.

The resemblance between this CRLB recursion and the recursion used in the KF should be noticed. For the linear Gaussian model, the CRLB recursion is exactly the same as the KF covariance matrix recursion implying that the Kalman filter is an efficient estimator. Also, the parametric and posterior CRLB in fact coincide for the special case of a linear Gaussian model. 



\section{Motion modeling}

Mathematical models of the dynamic behavior of the system as well as for the sensors are needed to be able to use the filtering techniques presented in Chapter 2 . In this chapter we therefore take a closer look at how the motion of a moving body can be modeled. The next chapter is dedicated for modeling the sensors.

There are several models of moving objects in litterature and an overview of some of them can be found in $[49,82]$. In this thesis we are interested in both the position and the orientation of an object moving in a three dimensional world. The theory of kinematics of a rigid body can provide a model of the expected behavior of such a system and will therefore be studied in this chapter. However, first it will be necessary to define a few coordinate frames in which the model can be described.

\subsection{Coordinate frames}

In order to describe the dynamic motion model, three coordinate systems have to be defined, one inertial, one that is fixed in the local environment and in which the tracking is performed, and one that is fixed in the moving rigid body.

- Inertial frame, $i$ : An inertial frame is a frame that is not subject to any accelerating forces. Its origin is fixed in the center of the earth and its axes are fixed relative to fix distant stars.

- Local environmental-fixed frame, $e$ : This system has its origin fixed in the local surroundings in which the rigid body (camera) is moving. A common setup is to have one of the coordinate axes aligned with gravity and the other two aligned with walls in the environment. This is the coordinate sys- 


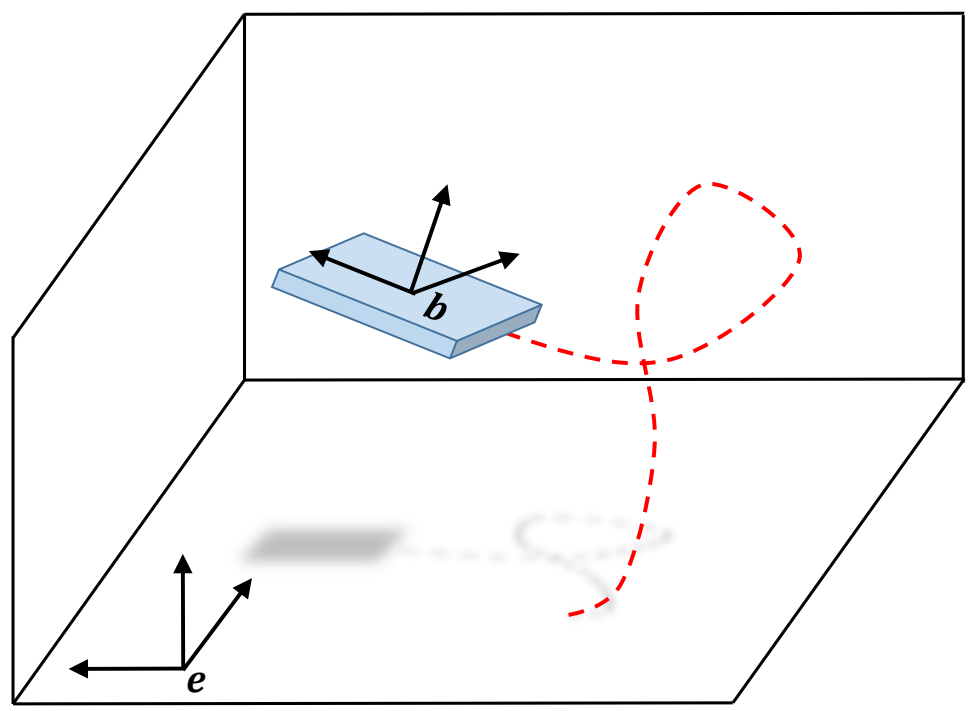

Figure 4.1: An illustration of the environmental-fixed, $e$, and the body-fixed, $b$, coordinate frames.

tem in which the tracking filter operates. Note that the environmental-fixed frame, strictly speaking, is not an inertial frame because of earth's orbital motion around the sun. Centrifugal and Coriolis forces are examples of fictitious accelerating forces on earth as a cause of earth not being an inertial frame.

- Body-fixed frame, $b$ : This system is fixed in the moving body. Its origin can be anywhere on the body. For a rigid body it is enough to know the orientation and position of one single point of the rigid body to be able to know the full pose of the whole rigid body $[16,90]$. In this thesis, the position and orientation of $b$ relative to $e$ is being tracked.

The coordinate frames are illustrated in Figure 4.1.

In this thesis, a subscript $f_{2} / f_{1}$ denotes "frame $f_{2}$ in relation to frame $f_{1}$ " and a superscript $f$ denotes "as given in frame $f$ " [82]. The vector $p_{b / e}^{e}$, for example, corresponds to the position of $b$ in relation to $e$ as given in $e$ and is one of the quantities that we are interested in tracking in this thesis.

\subsection{Translation}

In this thesis we track the position $p_{b / e}^{e}$ of a rigid body (a camera) moving in a local environment. The kinematic relation between position, $p$, velocity, $v$, and acceleration, $a$, is well known. Velocity and position can be obtained from inte- 
grating acceleration according to [90]

$$
\begin{aligned}
& \dot{p}_{b / e}^{e}(t)=v_{b / e}^{e}(t) \\
& \dot{v}_{b / e}^{e}(t)=a_{b / e}^{e}(t) .
\end{aligned}
$$

For example Euler forward or other numerical techniques [15] can be used to discretize (4.1). In this thesis, a discrete-time dynamic model is instead obtained by integrating between two time instances $t_{k-1} t_{k}$ assuming constant acceleration in the time interval. This gives [82]

$$
\begin{aligned}
& p_{b / e, k}^{e}=p_{b / e, k-1}^{e}+T_{k} v_{b / e, k-1}^{e}+\frac{T_{k}^{2}}{2} a_{b / e, k-1}^{e} \\
& v_{b / e, k}^{e}=v_{b / e, k-1}^{e}+T_{k} a_{b / e, k-1}^{e} .
\end{aligned}
$$

Here $T_{k}$ denotes the time passed between $t_{k-1}$ and $t_{k}$.

\subsection{Orientation}

Except for the position, the orientation of the body is also tracked in this thesis. Orientation can be mathematically described in many different ways. Examples of this are a triplet of rotation angles, for which there exist many different conventions, a $3 \times 3$ orthogonal rotation matrix, and a quadruple of unit quaternions $[16,21,38,76]$. Quaternions are continuous under integration and have no singular points in contrast to rotation angles. The quaternion representation is therefore the choice here. The quaternion $q_{b / e}$ such that $\left\|q_{b / e}\right\|_{2}=1$ corresponds to the rotation from $e$ to $b$.

The kinematics for quaternions might be less well known than that of position. Let

$$
\omega_{b / e}^{b}(t)=\left(\begin{array}{lll}
\omega_{x}(t) & \omega_{y}(t) & \omega_{z}(t)
\end{array}\right)^{T}
$$

be the angular velocity vector of $b$ relative to $e$ measured in $b$. The quaternion kinematics can then be expressed as [21, 45, 62]

$$
\dot{q}_{b / e}(t)=\left\{0, \frac{1}{2}\left(\omega_{b / e}^{b}(t)\right)^{T}\right\} \odot q_{b / e}(t)
$$

where $\odot$ corresponds to quaternion multiplication explained in for example [38]. There are similar expressions using $\omega_{b / e}^{e}(t)$ instead of $\omega_{b / e}^{b}(t)$. These are however not used in this thesis and are therefore omitted but can be found in [45].

Discretization of model (4.4) can, as mentioned in Section 4.2, be obtained with the Euler forward metod or other numerical techniques [15]. In this thesis we perform integration of (4.4) between $t_{k-1}$ and $t_{k}$ assuming constant angular velocity 
in the time interval. This give [62]

$$
\begin{aligned}
q_{b / e, k} & =q_{b / e, k-1} \odot \mathrm{e}^{\frac{T_{k}}{2}\left\{0,\left(\omega_{b / e, k-1}^{e}\right)^{T}\right\}} \\
& =q_{b / e, k-1} \odot\left\{\cos \left(\frac{T_{k}}{2}\left\|\omega_{b / e, k-1}^{b}\right\|_{2}\right), \frac{\left(\omega_{b / e, k-1}^{b}\right)^{T}}{\left\|\omega_{b / e, k-1}^{b}\right\|_{2}} \sin \left(\frac{T_{k}}{2}\left\|\omega_{b / e, k-1}^{b}\right\|_{2}\right\}\right) .
\end{aligned}
$$

Here $T_{k}$ denotes the time passed between $t_{k-1}$ and $t_{k}$. The last equality has been obtained by computing the quaternion exponent as explained in [62].

When the quantity $\frac{T_{k}}{2}\left\|\omega_{b / e, k-1}^{b}\right\|_{2}$ is small, which is the case either if the angular velocities are small or if the sampling interval $T_{k}$ is short, the expression (4.5) can be even further simplified. The small angle approximation states that $\cos \left(\frac{T_{k}}{2}\left\|\omega_{b / e, k-1}^{b}\right\|_{2}\right) \approx 1$ and that $\sin \left(\frac{T_{k}}{2}\left\|\omega_{b / e, k-1}^{b}\right\|_{2}\right) \approx \frac{T_{k}}{2}\left\|\omega_{b / e, k-1}^{b}\right\|_{2}$ giving

$$
\begin{aligned}
q_{b / e, k} & \approx q_{b / e, k-1} \odot\left(1, \frac{T_{k}}{2}\left(\omega_{b / e, k-1}^{b}\right)^{T}\right) \\
& =q_{b / e, k-1}+\frac{T_{k}}{2} S\left(\omega_{b / e, k-1}^{b}\right) q_{b / e, k-1}
\end{aligned}
$$

where the matrix $S$ is given by

$$
S\left(\omega_{b / e, k}^{b}\right)=\left(\begin{array}{cccc}
0 & -\omega_{x, k} & -\omega_{y, k} & -\omega_{z, k} \\
\omega_{x, k} & 0 & \omega_{z, k} & -\omega_{y, k} \\
\omega_{y, k} & -\omega_{z, k} & 0 & \omega_{x, k} \\
\omega_{z, k} & \omega_{y, k} & -\omega_{x, k} & 0
\end{array}\right)
$$

It should be noticed that, because of the approximation made, (4.6) does not guarantee that the quaternion keeps its unit length property over time, contrary to (4.5). Therefore other tricks, for example normalization, has to be used in combination with (4.6) in order to guarantee that the quaternion remains on the unit sphere.

\subsection{Combined translation and rotation}

Summarizing Section 4.2 and Section 4.3 gives the following model of a six degree of freedom rigid body moving in a three dimensional world

$$
\begin{aligned}
& p_{b / e, k}^{e}=p_{b / e, k-1}^{e}+T_{k} v_{b / e, k-1}^{e}+\frac{T_{k}^{2}}{2} a_{b / e, k-1}^{e} \\
& v_{b / e, k}^{e}=v_{b / e, k-1}^{e}+T_{k} a_{b / e, k-1}^{e} \\
& q_{b / e, k} \propto q_{b / e, k-1}+\frac{T_{k}}{2} S\left(\omega_{b / e, k-1}^{b}\right) q_{b / e, k-1} .
\end{aligned}
$$

Remember that the matrix $S\left(\omega_{b / e, k}^{b}\right)$ is given in (4.6d). Also note that $\propto$ has been used in $(4.7 \mathrm{c})$ to show that normalization may be nescessary for the quaternion to keep its unit lenghth. This model is still incomplete because, so far, the accel- 
eration $a_{b / e, k-1}^{e}$ and the angular velocity $\omega_{b / e, k-1}^{e}$ has not been modeled. There are some different approaches to this as explained in [82].

\subsubsection{Alternative 1: Model with additional states}

The first alternative is to model the acceleration and angular velocity as additional unknown system states,

$$
\begin{aligned}
a_{b / e, k}^{e} & =u_{k-1}^{a}+w_{k-1}^{a} \\
\omega_{b / e, k-1}^{b} & =u_{k-1}^{\omega}+w_{k-1}^{\omega} .
\end{aligned}
$$

The system inputs $u_{k}^{a}$ and $u_{k}^{\omega}$ corresponds the available information about the true acceleration and true angular velocity. This information can be obtained for example from prior knowledge about likely system behavior, from an internal system controller trying to regulate $a_{b / e, k}^{e}$ and $\omega_{b / e, k}^{e}$, or from measurements. The terms $w_{k-1}^{a}$ and $w_{k-1}^{\omega}$ corresponds to uncertainties in the information caused by for example system disturbances causing the system behavior to diverge from controller references and prior expectations, or it can correspond to the errors in acceleration and angular velocity measurements.

A special case of (4.8) is when there is no available information about true acceleration or true angular velocity. For this case $u_{k}^{a}$ and $u_{k}^{\omega}$ equals zero and the dynamics are modeled simply as noise.

\subsubsection{Alternative 2: Model with direct inputs}

A second alternative is to instead use the available information (4.8) about acceleration or true angular velocity as direct inputs to (4.7) without adding any new states to the model

$$
\begin{aligned}
& p_{b / e, k}^{e}=p_{b / e, k-1}^{e}+T_{k} v_{b / e, k-1}^{e}+\frac{T_{k}^{2}}{2}\left(u_{k-1}^{a}+w_{k-1}^{a}\right) \\
& v_{b / e, k}^{e}=v_{b / e, k-1}^{e}+T_{k}\left(u_{k-1}^{a}+w_{k-1}^{a}\right) \\
& q_{b / e, k} \propto q_{b / e, k-1}+\frac{T_{k}}{2} S\left(u_{k-1}^{\omega}+w_{k-1}^{\omega}\right) q_{b / e, k-1} .
\end{aligned}
$$

This approach has two advantages. The first is that the total number of system states become fewer leading to less computation time for the filters described in Chapter 2. The second is that there is a shorter time delay between obtaining measurements of acceleration and angular velocity and this information actually affecting the position and orientation estimates.

The model (4.9) is used in Paper A and B where IMU measurements are used as direct inputs to the model.

Now we have a models for the dynamic behavior of the system. The next chapter will introduce sensor models which relates system states $p_{b / e, k}^{e}, v_{b / e, k}^{e}$ and $q_{b / e, k}$ to the obtained measurements $y_{k}$. 



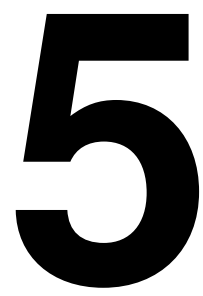

\section{Sensor modeling}

The filter framework presented in Chapter 2 allows for treating all measurements in the same way independently of their origin. The only requirement is that there is a mathematical description of how the sensor measurements relate to the system states. This opens up for easy fusion of measurements of different kinds. This is exploited in Paper A and B where camera, IMU and UWB are used together to obtain an accurate and mobile tracking system. In Paper $C$ the possibility to use also sound echoes is explored. These four different types of measurements and sensors will be described in this chapter. However, first sensor-specific coordinate frames have to be defined alongside the environmental- and body-fixed frames explained in Section 4.1.

\subsection{Sensor specific coordinate system}

In Section 4.1, the environmental-fixed and the body-fixed coordinate frames were introduced. Sensors however, in general operate in their own specific coordinate frames. A sensor-fixed coordinate frame, $s$, has its origin and its axes fixed relative to the sensor. Often a sensor is rigidly attached to the moving body, an assumption adopted in this thesis. In this case, $s$ is not only fixed relative to sensor but also relative to the rigid body itself and $b$. However, there is in general a translation and rotation separating the two frames from each as illustrated in Figure 5.1. The translation $r_{s / b}^{b}$ as observed from $b$, and rotation $R_{s / b}$, are constant parameters, which in this thesis are considered to be known. Calibration of these parameters is part of the preparatory work that has to be performed before using the filter. In this thesis, since we consider four different sensors, there are four different sensor-fixed frames $s_{\mathrm{IMU}}, s_{\mathrm{Cam}}, s_{\mathrm{UWB}}$, and $s_{\text {echo }}$ with corresponding four pairs of body to sensor translations and rotations. 


\subsection{Rotation matrices}

In the sensor models, the rotation between two frames are represented with a transformation matrix $R$ rather than with quaternions $q$, contrary to the motion model in Chapter 4. This is because now, the main purpose is to relate the measurements to the system states. Measurements therefore have to be transformed from $s$ to $e$, in which the filter is operating and the system states are expressed. For this, the transformation matrix representation is convenient since a change of basis of some vector $v$ is easily done according to

$$
v^{e}=R_{e / s} v^{s}=R_{s / e}^{T} v^{s},
$$

Recall from Section 4.1 that a subscript $s / e$ means "frame $s$ in relation to frame $e$ " and that a superscript $e$ means "as given in frame $e$ " [82].

It should also be notices that (5.1) can be written as

$$
v^{e}=R_{s / b} R_{b / e, k}^{T} v^{s} .
$$

where $R_{s / b}$ is the pre-calibrated orientation of $s$ relative to $b$ while $R_{b / e, k}$ is the time varying orientation of the body in the local environment. Conversion between the quaternion representation $q_{b / e, k}$ used in the motion model in Section 4 and the matrix representation $R_{b / e, k}$ used here is easily done according to [21]

$$
\begin{aligned}
& R_{b / e, k} \triangleq\left(\begin{array}{lll}
r_{11, k} & r_{12, k} & r_{13, k} \\
r_{21, k} & r_{22, k} & r_{23, k} \\
r_{31, k} & r_{31, k} & r_{33, k}
\end{array}\right) \triangleq R_{b / e, k}\left(q_{b / e, k}\right) \\
& =\left(\begin{array}{ccc}
2 q_{0, k}^{2}+2 q_{1, k}^{2}-1 & 2 q_{1, k} q_{2, k}-2 q_{0, k} q_{3, k} & 2 q_{1, k} q_{3, k}+2 q_{0, k} q_{2, k} \\
2 q_{1, k} q_{2, k}+2 q_{0, k} q_{3, k} & 2 q_{0, k}^{2}+2 q_{2, k}^{2}-1 & 2 q_{2, k} q_{3, k}-2 q_{0, k} q_{1, k} \\
2 q_{1, k} q_{3, k}-2 q_{0, k} q_{2, k} & 2 q_{2, k} q_{3, k}+2 q_{0, k} q_{1, k} & 2 q_{0, k}^{2}+2 q_{3, k}^{2}-1
\end{array}\right) \\
& q_{b / e, k} \triangleq\left\{q_{0, k} \quad q_{1, k} \quad q_{2, k} \quad q_{3, k}\right\} \triangleq q_{b / e, k}\left(R_{b / e, k}\right) \\
& =\left\{\frac{\sqrt{1+r_{11, k}+r_{22, k}+r_{33, k}}}{2} \frac{r_{32, k}-r_{23, k}}{2 \sqrt{1+r_{11, k}+r_{22, k}+r_{33, k}}} \quad \frac{r_{13, k}-r_{31, k}}{2 \sqrt{1+r_{11, k}+r_{22, k}+r_{33, k}}} \frac{r_{21, k}-r_{12, k}}{2 \sqrt{1+r_{11, k}+r_{22, k}+r_{33, k}}}\right\} .
\end{aligned}
$$

Note that caution has to be taken in the implementation of (5.3c) because numerical instability occurs when $q_{0, k}$ is small.

The transformation (5.1) can also be done using quaternion operations [62]. However, the transformation matrix representation makes it more straightforward to derive the Jacobians needed in an EKF, Section 2.4.1, and to compute the parametric CRLB, Section 3.2.2.

\subsection{Inertial measurement unit}

In this thesis we will use six degree of freedom micro-electro-mechanical system (MEMS) inertial measurement units (IMU:s) measuring acceleration and angular 


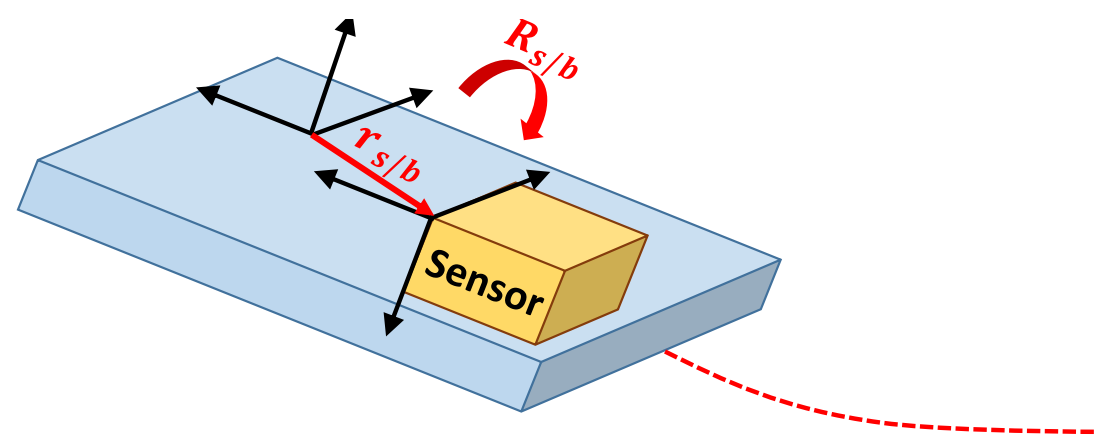

Figure 5.1: Illustration of the body-fixed, $b$, and the sensor-fixed, $s$, coordinate frames and the translation $r_{s / b}$ and rotation $R_{s / b}$ relating them to one another.

velocity. This is a type of sensor commonly used for indoor localization and navigation. They have the advantages of being small and light, have relatively small power consumption and are relatively cheap. They are self-sustainable and do not make use of any external infrastructure, contrary to for example GPS, wireless radio receivers, camera or similar.

\subsubsection{Measurement model}

The coordinate frame in which the IMU is operating will be denoted $s_{\mathrm{IMU}}$. An IMU measures the specific forces, inertial acceleration $a_{S_{\mathrm{IMU}} / i, k}$ along with gravity $g$, and angular velocity, $\omega_{S_{\mathrm{IMU}} / i, k}$, of the sensor itself relative to the inertial coordinate frame $i$. In practice the measurements are not exact but perturbed by measurement errors leading to the following model [125],

$$
\begin{aligned}
& y_{k}^{a}=a_{s_{\mathrm{IMU}} / i, k}^{s_{\mathrm{IMU}}}+g^{s_{\mathrm{IMU}}}+e_{k}^{a} \\
& y_{k}^{\omega}=\omega_{S_{\mathrm{IMU}} / i, k}^{s_{\mathrm{IMU}}}+e_{k}^{\omega} .
\end{aligned}
$$

Here $e_{k}^{a}$ and $e_{k}^{\omega}$ corresponds to the measurement errors.

An environmental-fixed frame on earth is, as mentioned before, strictly speaking not an inertial frame. However, the difference is small. It is therefore common to consider earth-fixed frames, such as $e$, as approximately inertial, knowing it is just an approximation. This approximation leads to the simplified model

$$
\begin{aligned}
& y_{k}^{a}=a_{S_{\mathrm{IMU}} / e, k}^{s_{\mathrm{IU}}}+g^{s_{\mathrm{IMU}}}+e_{k}^{a}, \\
& y_{k}^{\omega}=\omega_{S_{\mathrm{IMU}}}^{S_{\mathrm{IMU}} / e, k}+e_{k}^{\omega},
\end{aligned}
$$

where all effects caused by earth's orbit around our sun is neglected.

As can be seen in (5.4) and (5.5), the measurements are given along the three axes of the IMU-fixed coordinate frame $s_{\mathrm{IMU}}$. It is for practical reasons convenient to choose $b$ such that it coincides with $s_{\text {IMU }}$. In that case, lever arm effects are not 
present when transforming the measurements of the IMU motion into measurements of the motion of the body,

$$
\begin{aligned}
y_{k}^{a} & =a_{b / e, k}^{b}+g^{b}+e_{k}^{a}=R_{b / e, k}\left(a_{b / e, k}^{e}+g^{e}\right)+e_{k}^{a} \\
y_{k}^{\omega} & =\omega_{b / e, k}^{b}+e_{k}^{\omega} .
\end{aligned}
$$

A more accurate model including an inertial frame and lever arm effects is discussed in Paper A.

The second equality in (5.6a) is expressed as the acceleration in the earth frame rather than in the IMU or body frame. It relates the measurements to the system states $p_{b / e, k}^{e}, v_{b / e, k}^{e}$ and $r_{b / e, k}$ (qr equivalently $R_{b / e, k}$ as used here). the gravity $g^{e}$ as measured in the local environment is assumed to be a known model parameter.

Note that the measurements (5.6) now can be rewritten as

$$
\begin{aligned}
a_{b / e, k}^{e} & =R_{b / e, k}^{T}\left(y_{k}^{a}-e_{k}^{a}\right)-g^{e} \\
\omega_{b / e, k}^{b} & =y_{k}^{\omega}-e_{k}^{\omega}
\end{aligned}
$$

and used as direct inputs to the motion model, as described in Section 4.4, with

$$
\begin{aligned}
& u_{k-1}^{a} \triangleq R_{b / e, k}^{T} y_{k}^{a}-g^{e} \\
& w_{k-1}^{a} \triangleq-R_{b / e, k}^{T} e_{k}^{a} \\
& u_{k-1}^{a} \triangleq y_{k}^{\omega} \\
& w_{k-1}^{\omega} \triangleq-e_{k}^{\omega}
\end{aligned}
$$

\subsubsection{Model errors}

The two main contributors to the additive measurement errors $e_{k}^{a}$ and $e_{k}^{\omega}$ are [135]:

- Additive white noise due to, for example, thermo-mechanical noise with a correlation time much shorter than the sampling time of the sensor.

- Additive measurement bias. It is common to model the bias in two parts, a constant part and a part that changes over time. The constant bias is technically not constant. It is modeled as constant during the run-time of the IMU but can change each time the IMU is turned off and on because of different effects such as changes in the physical properties of the sensor or changes in the initial conditions of the internal signal processing. The time variations in the bias during run-time are due to for example flicker noise in the sensor hardware. The in-run dynamics of the bias is usually modeled as a random walk process.

The Allan variance, data variance computed using different window sizes, is a commonly used tool for analyzing the additive error terms. It can be used to analyze both the type of noise that the error terms are composed of, and the error variance [28]. 
Except for additive noise there are also several other error sources in an IMU such as nonlinearities in the hardware, gain offsets, alignment errors in the IMU axis, g-sensitivity ${ }^{1}$ and cross-talk between the channels [6]. It is however possible to, more or less, calibrate for these types of errors. This is for example done by the IMU manufacturer for more accurate and expensive IMU:s or done by the user once before staring to use the IMU. The references $[34,64,116]$ give suggestions of different calibration techniques. The models presented in Section 5.3.1 are, after sensor calibration, often sufficiently good even though they do not include any nonadditive error sources.

Inertial measurements can be used to dead reckon a pose. However, as the measurements are integrated over time, the errors accumulate. The drift can be reduced if the measurement bias can be estimated and compensated for but even bias compensation does not completely eliminate the drift. The strength of an IMU is in its short term predictions. It is therefore often used in hybrid systems together with other types of sensors if there is a need for tracking over longer time periods. In this thesis, a camera-IMU system is considered in Paper A and a camera-IMU-UWB system is used inPaper B.

\subsection{Optical camera}

In order to be able to use images for tracking purposes, features has to be found, recognized, and associated to a point in the real world or a map of the real world. Computer vision techniques such as feature extraction, feature identification, and feature association are common tools for this. Harris corners [54], SIFT [85] and SURF [9] features are commonly used image features. RANSAC [31] is a relatively old but still commonly used feature association and outlier detection algorithm but there is an abundance of other choices as well [121]. Image processing often requires more memory and computational resources than other signal processing. This limits which image processing methods that can be used in a real-time application and how fast new processed image measurements can be fed to a tracking filter. However, a camera is a rich sensor with the possibility to provide a user with much information if the image processing is done wisely.

\subsubsection{Measurement model}

It is crucial to have a model of how a camera projects our three dimensional world onto a two dimensional image sensor when using detected image features in a filter. The most commonly used camera model for tracking purposes is the pinhole camera model, which is illustrated in Figure 5.2. There it can be seen that the camera-fixed frame $s_{\text {cam }}$ is fixed in the optical center of the camera and that the image sensor is at a distance $f$, the focal distance, from the optical center.

Introduce the notation $l^{m} \triangleq\left(\begin{array}{lll}l_{x}^{m} & l_{y}^{m} & l_{z}^{m}\end{array}\right)^{T}$ for the vector between the camera

\footnotetext{
${ }^{1} \mathrm{G}$-sensitivity means that the gravitation has influence not only on the acceleration measurements but also on the angular velocity measurements.
} 
and a point $m$ in the three dimensional world as observed by the camera. This vector is illustrated in Figure 5.2b for clarity. The image coordinates, at which the corresponding image $\widetilde{m}$ of $m$ appears, are then according to the pinhole model [121]

$$
\begin{aligned}
& \tilde{l}_{x}^{\widetilde{m}}=f \frac{l_{x}^{m}}{l_{z}^{m}}=\frac{l_{x}^{m}}{l_{z}^{m}} \\
& \tilde{l}_{y}^{\widetilde{m}}=f \frac{l_{y}^{m}}{l_{z}^{m}}=\frac{l_{y}^{m}}{l_{z}^{m}}
\end{aligned}
$$

under the assumption that $f=1$. This can be realized by studying similar triangles in Figure 5.2b. The image coordinates $\tilde{l}_{x}^{\widetilde{m}}$ and $\tilde{l}_{y}^{\widetilde{m}}$ in (5.12) are sometimes referred to as the normalized image coordinates because of the assumption made on the focal length.

The vector $\tilde{l}^{\tilde{m}}$ in Figure $5.2 \mathrm{~b}$ corresponds to the vector from the optical center to the projected point $\widetilde{m}$ in the image as observed by the camera. This vector can be expressed in terms of the image coordinates (5.12) according to $\tilde{l}^{\widetilde{m}} \triangleq$

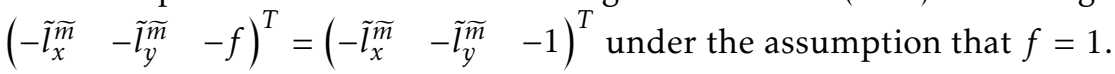

An alternative but equivalent pinhole model is shown in Figure 5.2c. Here the image is instead formed in front of the optical center which leads to a model where the image is not formed upside down and the elements of the vector $\tilde{l}^{\widetilde{m}}$ has no minus signs according to $\tilde{l}^{\tilde{m}}=\left(\begin{array}{lll}\tilde{l}_{x}^{\widetilde{m}} & \tilde{l}_{y}^{\widetilde{m}} & 1\end{array}\right)^{T}$. This can not be implemented for a real pinhole camera where the image is always formed at the back of the camera, but the modeling of a virtual image in front of the camera simplifies the model slightly since the virtual image is formed in an upright position rather than upside down.

The projection (5.12) can be used to relate image coordinates of a detected and identified image feature $\widetilde{m}$ to the states $p_{b / e, k}^{e}, v_{b / e, k}^{e}, q_{b / e, k}$ (or alternatively $R_{b / e, k}$ as used here) in (4.9). This is done through the relation

$$
l_{k}^{m}=R_{s_{\mathrm{cam}} / b}\left(R_{b / e, k}\left(p_{m / e}^{e}-p_{b / e, k}^{e}\right)-r_{s_{\mathrm{cam}} / b}^{b}\right)
$$

where vector decomposition of $l^{m}$ has been used. In (5.13a), $p_{m / e}^{e}$ corresponds to the position of the corresponding landmark $m$ as give in $e$. This is a model parameter similar to $r_{s_{\mathrm{cam}} / b}^{b}$ and $R_{s_{\mathrm{cam}} / b}$, the pre-calibrated translation and rotation between the body frame and the camera frame. Note the extra time index $k$ on $l^{m}$ in (5.13a) introduced because of the relation to the time-varying states.

With (5.12) and (5.13a) the following camera measurement model can be formed:

$$
y_{i, k}^{\mathrm{cam}}=\left(\begin{array}{c}
\tilde{l}_{x, k}^{\tilde{m}_{i}} \\
\tilde{l}_{y, k}^{m_{i}}
\end{array}\right)+e_{i, k}^{\mathrm{cam}}=\left(\begin{array}{c}
\frac{l_{x, k}^{m_{i}}}{l_{z, k}^{m_{i}}} \\
l_{y, k}^{m_{i}} \\
l_{z, k}^{m_{i}}
\end{array}\right)+e_{i, k}^{\mathrm{cam}}
$$

where $e_{i, k}^{\text {cam }}$ corresponds to the measurement errors. Note that several landmarks 


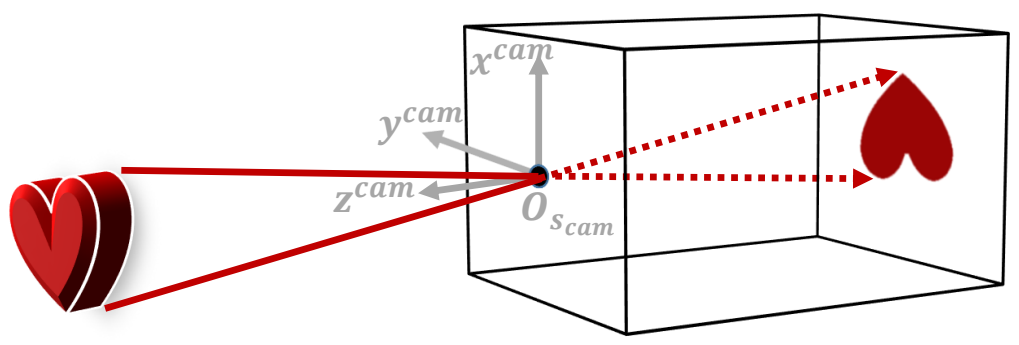

(a)

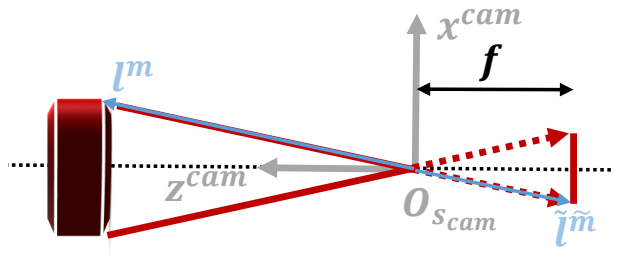

(b)

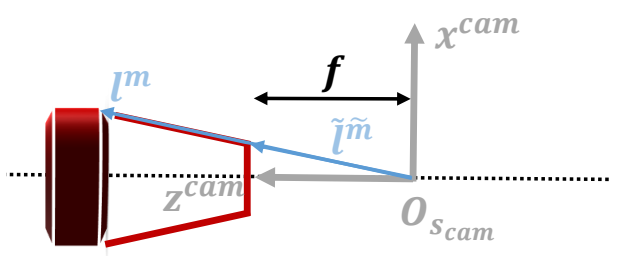

(c)

Figure 5.2: Illustration of a pinhole camera and the pinhole camera projection. a) The pinhole camera principle. $\boldsymbol{b}$ ) The pinhole camera projection model. c) An alternative pinhole camera projection model where a the image is formed in front of the optical center rather than at the back of the camera.

can be present in one image frame, thereof the extra index $i$ in (5.13b) denoting the landmark $m_{i}$. Note also that, even though several landmarks can be in the image at the same time, not all landmarks must be present at all times $t_{k}$. The pose of the camera and its field of view limits which landmarks are visible in the image.

The distance information $l_{z, k}^{m_{i}}$ gets lost in the camera projection, as can be seen in (5.13b). This type of camera measurements therefore in fact corresponds to angleof-arrival measurements (see Section 5.5 for a description of angle-of-arrival measurements).

The positions $p_{m_{i} / e}^{e}$ of the landmarks in (5.13a) can be treated in different ways. They can be treated as known parameters, in which case they have to be mapped beforehand similar to $r_{s_{\mathrm{cam}} / b}^{b}$ and $R_{s_{\mathrm{cam}} / b}$. They can also be treated as unknown states in an augmented system state vector, in which case they are estimated in a SLAM sense as described in Section 2.5. In the simulation study in Paper A it is assumed that the position of the landmarks are known while the second alternative is adopted in Paper B. 


\subsubsection{Model errors}

The camera model explained in Section 5.4.1 is a very simplified model of a real camera. For one, the focal length was assumed to be 1 . Other factors, such as lens distortions, defocus and pixelation at the image sensor are also neglected [121]. In order to account for defocus in images a camera model with a lens has to be used. Paper A presents model based on ray tracing through a thick lens.

A camera can to some degree be calibrated to better fit the normalized pinhole model (5.13). The reference [139] provides an example of this and gives references to several more. The camera calibration problem has been extensively researched and there are several available code libraries, for example the OpenCv calib3d library [1] or its Matlab implementation, the Camera Calibration Toolbox for Matlab [14]. For a historical overview of the development of camera calibration methods we refer to [19].

Different distortion models are of course suitable for different types of lenses. Here, as an example, we present the model used in the Camera Calibration Toolbox for Matlab [14] inspired by work presented in [25, 39, 56] among others. The following intrinsic camera parameters are used to to model the lens and the lens distortions:

Focal length - one focal length parameter for each image dimension, $f=\left(\begin{array}{ll}f_{x} & f_{y}\end{array}\right)^{T}$.

Principal point - the center of the image sensor in case it is not centred exactly behind the optical center. One parameter for each image axis, $c=\left(\begin{array}{ll}c_{x} & c_{y}\end{array}\right)^{T}$.

Skew coefficient - the angle between pixel rows and pixel columns in the image sensor in case the pixels are not exactly orthogonally aligned, $\alpha$.

Distortion coefficient - radial and tangential lens distortion parameters, $k=\left(\begin{array}{lllll}k_{1} & k_{2} & k_{3} & k_{4} & k_{5}\end{array}\right)^{T}$.

The camera projection can then be computed according to

$$
\begin{aligned}
& \tilde{l}_{x}=f_{x}\left(x+\alpha y+c_{x}\right) \\
& \tilde{l}_{y}=f_{y}\left(y+c_{y}\right)
\end{aligned}
$$

where

$$
\begin{aligned}
& x=\left(1+k_{1} r^{2}+k_{2} r^{4}+k_{5} r^{6}\right) \frac{l_{x}}{l_{z}}+2 k_{3} l_{x} l_{y}+k_{4}\left(r^{2}+2 l_{x}^{2}\right) \\
& y=\left(1+k_{1} r^{2}+k_{2} r^{4}+k_{5} r^{6}\right) \frac{l_{y}}{l_{z}}+2 k_{4} l_{x} l_{y}+k_{3}\left(r^{2}+2 l_{y}^{2}\right)
\end{aligned}
$$

and where

$$
r^{2}=l_{x}^{2}+l_{y}^{2}
$$


The indices $m_{i}$ and $\widetilde{m}$ and $k$ on $l$ and $\tilde{l}$ has been left out from (5.14) for simpler notation.

Once a camera has been calibrated and the intrinsic parameters are found, images can be corrected for the distortion in (5.14) such that corrected image coordinates corresponds better to the normalized image coordinates in the model in Section 5.4.1.

It is hard to say something general about the nature of the additive measurement errors $e_{i, k}^{\mathrm{cam}}$ in (5.13b) due to the complex nature of cameras and the variety of available computer vision and image processing tools. Also, this is a subject not that well researched and litterature mostly presents experimental results rather than theoretical studies. The work presented in [104] is an exception. Experimental results are difficult to transfer to environments and scenarios that differ from the experimental setup. A common choice in tracking applications is therefore to simply model the errors as additive Gaussian noise.

\subsection{Ultra wide band}

Localization and tracking based on transmission and receiving of radio signals has been researchedand executed for many decades by now. In this thesis we consider a setup with one mobile receiver or master unit and several stationary transmitters or slave units deployed in the local environment, as illustrated in Figure 5.3. There are several different techniques and types of measurements for radio based tracking systems. Some examples taken from $[83,114]$ are:

- Received-signal-strength methods: The power of the transmitted radio signal decays with the travelled distance. Measuring the power of the received signal can hence provide information about how far away a receiver is from a stationary transmitter. The information carried in received-signalstrength measurements are illustrated in Figure 5.3a.

- Time-based methods: The propagation speed of radio signals in different media is well known in physics. Hence measuring the time it takes for a transmitted signal to reach a receiver provides information about the distance between the two devices, similar to received-signal-strength measurements. The information carried in time-based measurements is illustrated in Figure 5.3a.

- Angle-based methods: By using arrays of receivers, information about the direction of an incoming radio signal can be extracted. This gives information about in which directions the stationary transmitters can be found, as illustrated in Figure 5.3b.

- Fingerprinting methods: These techniques are based on the assumption that each limited set of nearby locations has its own unique radio signal fingerprint. Such a fingerprint can for example consist of a number of detectable radio signal transmitters, the identifiers of the detectable transmit- 


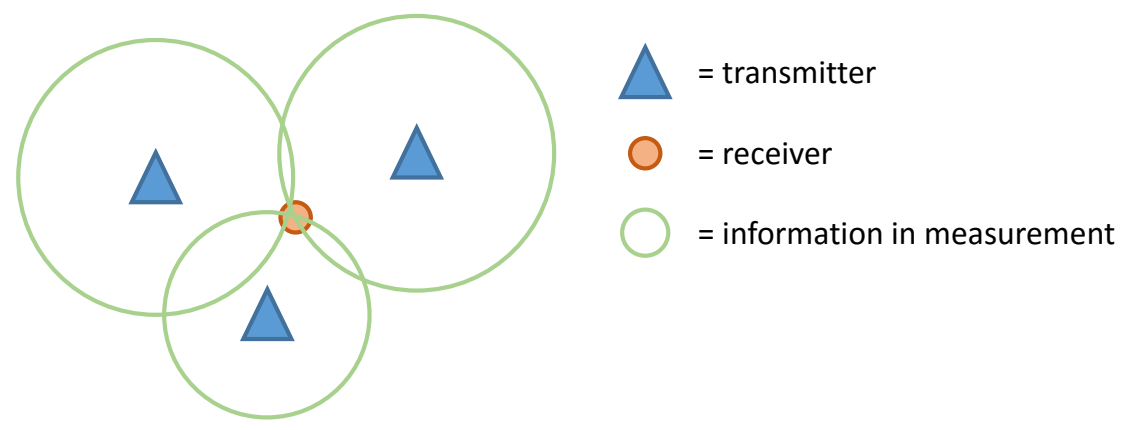

(a) Lateration: For example received-signal-strength or time-based measurements contain information about the distance from stationary transmitters with known positions to the mobile receiver.
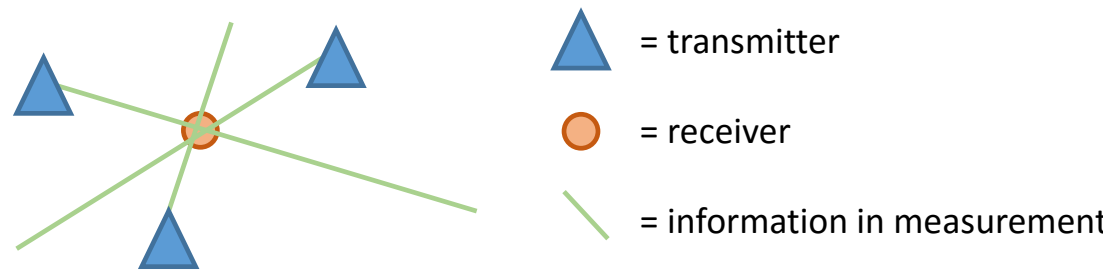

(b) Angulation: For example measurements from receiver arrays can contain information about the direction in which the mobile can find a stationary transmitter.

Figure 5.3: Illustration of the lateration and angulation principles. Measurements from several stationary transmitters can be combined to determine the position of a mobile receiver.

ters, received signal strength of the detectable transmitters, or similar. If this fingerprint can be recognized, then the current location of the receiver is recognized at the same time.

As seen above, fingerprinting methods can provide direct estimates of position, whereas received signal strength, time-based and angle-based methods only provide information about how far away a transmitter is or in which direction it can be found. However, if the location of the stationary transmitters are known, lateration and angulation techniques $[2,83]$ can be used in order to obtain position estimates, see Figure 5.3 for an illustration. None of the above methods provides information about the full pose of the receiver, but only about the position. Fusion with some other type of sensor is therefore necessary to obtain tracking of full pose.

Ultra wide band (UWB) signals are a type of radio signals with a band with broader than that of many others. A UWB signal is defined as a radio signal with a bandwidth greater than $20 \%$ of its center frequency, or with a bandwidth greater than 
$500 \mathrm{MHz}$. UWB signals are typically short pulses with a spectrum that looks much like white noise and are only allowed to be transmitted with a very low power. The UWB technology therefore only has negligible influence over other radio services, which can hence be running simultaneously.

A good overview of UWB and UWB-based localization is given in [41]. Thanks to the short pulse technology, time-based range measurements as described above can be obtained with high accuracy of decimeters down to centimeters. Also, the UWB technology is better at overcoming the multipath problem than other more traditional radio technologies and has been used for see-through-wall applications [136, 141]. These properties make the technology suitable for high accuracy indoor tracking. Time-of-flight measurements is therefore the choice in Paper B, where only a camera and an IMU was not enough because of problems with error drift.

\subsubsection{Measurement model}

Time-of-flight $\tau$ between a radio transmitter $m_{i}$ and a radio receiver attached to the moving rigid body is related to the distance between the units according to

$$
\tau_{i}=\frac{\left\|l^{m_{i}}\right\|_{2}}{c} .
$$

Here $l^{m_{i}}$ is the vector from the receiver to the transmitter and $c$ is the propagation speed of the signal. Note that here it is not important in which frame $l^{m_{i}}$ is measured because its norm is the same in any frame. For simplicity we choose to express $l^{m_{i}}$ in the $e$ frame. This means that $l^{m_{i}}$ can be decomposed and expressed in the system model states $p_{b / e, k}^{e}, v_{b / e, k}^{e}$ and $q_{b / e, k}$ (or equivalent $R_{b / e, k}$ as used here) in (4.4.2) according to

$$
l_{k}^{m_{i}}=p_{m_{i} / e}^{e}-p_{b / e, k}^{e}-r_{s_{\mathrm{UWB}} / b}^{e}=p_{m_{i} / e}^{e}-p_{b / e, k}^{e}-R_{b / e, k}^{T} r_{s / b}^{b} .
$$

Here $p_{m_{i} / e}^{e}$ is the position of the transmitter $m_{i}$ as observed from $e$. Also, recall that $r_{s_{\mathrm{UWB}} / b}^{b}$ is the pre-calibrated translation from $b$ to $s_{\mathrm{UWB}}$. Note the extra time index $k$ on $l^{m_{i}}$ in (5.16) because of the introduction of the relation to the timevarying states.

With help of (5.15) and (5.16) it is possible to obtain the following measurement model:

$$
y_{i, k}^{\mathrm{abbrUWB}}=\tau_{i}+e_{i, k}^{\mathrm{UWB}}=\frac{\left\|p_{m_{i} / e}^{e}-p_{b / e, k}^{e}-R_{b / e, k}^{T} r_{s / b}^{b}\right\|_{2}}{c}+e_{i, k}^{\mathrm{UWB}}
$$

It should be noted that the rotation parameter $R_{s_{\mathrm{UWB}} / b}$ is in fact not relevant in this model. Only $r_{s_{\mathrm{UWB}} / b}^{b}$ has to be calibrated.

Performing positioning with lateration methods requires measurements from several transmitters. This means that lateration algorithms have to wait for several measurements to arrive before they can be executed. An advantage of using the time-of-flight of measurements in a filter instead of using lateration techniques is 
that the measurements can be fed to the filter one and one as they arrive, leading to less estimation delay.

The positions $p_{m_{i} / e}^{e}$ of the transmitters in (5.17) can be treated in different ways. They can be treated as known parameters, in which case they have to be mapped beforehand similar to $r_{s_{\mathrm{UWB}} / b}^{b}$. They can also be treated as unknown states in an augmented system state vector, in which case they are estimated in a SLAM sense as described in Section 2.5.

\subsubsection{Model errors}

Time-of-flight measurements obtained with UWB technology can be obtained in different ways, using one-way or two-way ranging schemes. How it is done have impact on the model errors.

In a one-way scheme, there is a clear transmitter-receiver relationship between the involved units and the signal is only sent one way, from the transmitter to the receiver. Time-of-flight measurements can only be obtained from these one-way transmissions if the units are time synchronized, or if the relative clock delay is known or estimated. Time synchronization errors or error in the estimated clock delay will give arise to measurement bias. Also relative clock drift is a problem in one-way schemes. More details about both these errors types can be found in [22].

In a two-way scheme, both the two units can transmit and receive signals. It is then more informative to talk about a mobile master and stationary slave units rather than receiver and transmitter units. A master unit initiates the measuring of time delay by transmitting a message to the slave unit. The slave unit then responds to this message. There are different ways in which a slave unit can respond resulting in different methods for computing time-of-flight $[11,22]$ :

Alternative 1 The slave unit replies immediately when receiving a signal from the master. The master can time the total time-of-flight back and forth for the signal and half the computed time corresponds to the one-way distance. Note that the master needs no information about the clock of the slave for the measurement to be accurate using this technique. A problem with this method though is that there is some processing time in the slave unit before it can reply. This processing time will enter the measurements in the same way as the clock synchronization error. The problem can be solved if the slave times its own processing time and send this information back in the echoed signal so that the master can subtract it from the total time-of-flight before computing the one way time-of-flight as illustrated in Figure 5.4a. However, even though this means that no time synchronization is needed, there is still a problem with relative clock drift in the two units that can not be completely ignored.

Alternative 2 Another alternative is to let the slave measure its own processing time and reply twice with the same delay. The first device can then itself measure the processing time of the slave by measuring the time between 


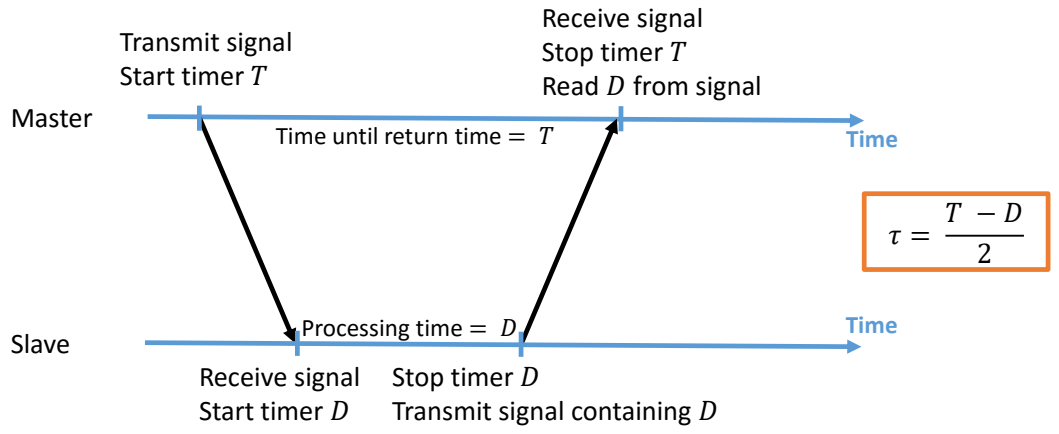

(a) Two way ranging alternative 1: The slave unit measures its own processing time and sends this information to the master with the echoed signal.

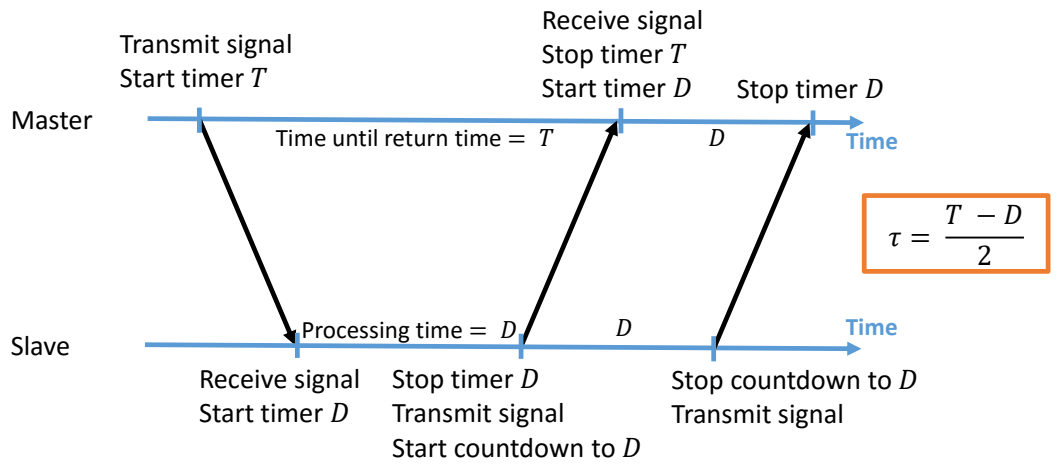

(b) Two way ranging alternative 2: The slave unit echos the master unit twice such that the master can compute the processing time of the slave by itself.

Figure 5.4: Illustration of different two-way ranging methods for obtaining time-of-flight information $\tau$.

the two replies as illustrated in Figure 5.4b. This method should, in theory, not suffer from time synchronization errors nor errors due to relative clock drift or unknown processing time in the slave unit.

Another major source of error, except for clock related errors, are non-line-ofsight or multipath conditions [22]. Multipath propagation causes interference of signals arriving at the receiver via different propagation paths. With UWB it is possible to resolv the multiple paths $[17,134]$ but it is a difficult nontrivial problem. Non-line-of-sight means that the shortest path between the receiver and the transmitter is blocked. This can cause the measurements to be biased and the obtained time delay measurements to in average be too long.

Attempts have been made to model the additive measurement error term $e_{i, k}^{\mathrm{UWB}}$ 
in (5.17). Examples of this are [3, 100], where error PDF such as the log-normal and the skew-t distributions are explored. What both these probability density models have in common is long heavy tails for positive errors but shorter tails for negative errors reflecting the problem with non-line-of-sight. In Paper B a non-line-of-sight outlier detector based on measurement predictions made from IMU measurements was used in order to reduce harmful use of inaccurate UWB measurements.

\subsection{Detected known signals}

Some types of sensors, such as radars and sonars, work by analyzing echoes of wave signals that they themselves have transmitted and that have been reflected in an object or surface in the environment. The time-of-flight between transmission and return of the signal gives information about the distance to the reflecting object as described in Section 5.5. However, Doppler shift in the returned signal can also give additional information about the relative motion of the transmitter and the reflecting object [99].

Radars and sonars are typical examples of sensors that extract range and velocity information from reflected signals. These sensors have however traditionally been used in for example marine and flight applications. They have traditionally not been used for small-scale or indoor localization and navigation. Examples of sensors relying on reflected signals, that have been traditionally used an environments of smaller scale, are lidars and ultrasonic sensors. However, it is not common to extract any Doppler information from these sensors. They usually provide only range measurements. Paper $C$ explores the possibility to extract both range and velocity information from reflected signals in indoor environments.

There are suggestions in literature how to extract the time delay and Doppler information from reflected signals:

- Pulse-Doppler signal processing, where the idea is to split the signal into shorter intervals. In each interval, the fast Fourier transform is computed and the peaks are detected. The phase shift at the peak frequency from one interval to the next is proportional to the time delay. The references $[108,122]$ exemplify this technique.

- Frequency division, where the signal can be split into a base band signal and the carrier signal. Then, the time scale is estimated from the carrier frequency, and the time shift from the base band signal, e.g., using correlation analysis (where the time scale can be neglected). This technique is exemplified in [44].

- Matched filters, where the known signal waveform is reversed, with different time scales and time delays, and applied as a matched filter. The maximum of the resulting two-dimensional function, the ambiguity function, corresponds to the sought delay and scale. In practice this can be seen 
as an exhaustive search on a $2 \mathrm{D}$ grid. The references $[43,97]$ exemplify this technique.

\subsubsection{Measurement model}

A reflected wide-band wave signal can, in the time domain, be modeled as [68]

$$
s^{\mathrm{echo}}(t)=\alpha s(\beta(t-\tau)) .
$$

Here $s(t)$ is the transmitted, usually known, signal. The parameter $1 / \beta$ corresponds to the Doppler stretch factor, $\tau$ is the time-of-flight and $\alpha$ is the attenuation due to path losses. In the frequency domain the corresponding model is

$$
S^{\text {echo }}(f)=\frac{1}{|\beta|} S\left(\frac{f}{\beta}\right) \mathrm{e}^{-i 2 \pi \tau f} .
$$

In this thesis it is assumed that the sensor is attached to a moving body and that the environment and its reflecting objects are stationary. The sensor-fixed frame $s_{\text {echo }}$ is attached in the signal receiver of the sensor. The parameters $\tau$ and $1 / \beta$ contains information about the distance to the reflecting object as well as the radial velocity relative to the reflecting object [99].

Let $l^{m_{i}}$ denote the vector from $s_{\text {echo }}$ to the reflecting object $m_{i}$, as given in $e$. The distance between the sensor and the reflecting object then corresponds to $\left\|l^{m_{i}}\right\|_{2}$. Let also $v^{m_{i}}$ denote the velocity of the $s_{\text {echo }}$ relative to $e$, in which the objects $m_{i}$ are stationary. The radial velocity $v_{r}^{m_{i}}$ can be obtained by projection of $v^{m_{i}}$ onto $l^{m_{i}}$

$$
v_{r}^{m_{i}}=v^{m_{i}} \cdot \frac{l^{m_{i}}}{\left\|l^{m_{i}}\right\|_{2}} .
$$

See Figure 5.5 for a clarifying illustration of $l^{m_{i}}, v^{m_{i}}$ and $v_{r}^{m_{i}}$.

A connection to the states $p_{b / e, k}^{e}, v_{b / e, k}^{e}$ and $q_{b / e, k}$ (or equivalent $R_{b / e, k}$ as used here) in (4.9) can be obtained from the two following equations:

$$
l_{k}^{m_{i}}=p_{m_{i} / e}^{e}-p_{b / e, k}^{e}-r_{s_{\mathrm{echo}} / b}^{e}=p_{m_{i} / e}^{e}-p_{b / e, k}^{e}-R_{b / e, k}^{T} r_{s_{\mathrm{echo}} / b}^{b}
$$

Here vector decomposition has been used. $p_{m_{i} / e}^{e}$ corresponds to the position of the reflecting object $m_{i}$ as observed in $e$.

$$
v_{k}^{m_{i}}=v_{b / e, k}^{e}+\omega_{b / e, k}^{e} \times r_{s_{\mathrm{echo}} / b}^{e}=v_{b / e, k}^{e}+R_{b / e, k}^{T} \omega_{b / e, k}^{b} \times r_{s_{\mathrm{echo}} / b}^{b}
$$

Lever arm effects have to be accounted for because the origins of $b$ and $s_{\text {echo }}$ may not be placed in the same point. This is done using the term $\omega_{b / e, k}^{b} \times r_{s_{\text {echo }} / b}^{b}$ in (5.21b). This introduces $\omega_{b / e, k}^{b}$ into the equation. Recall from Section 5.3 that this is the angular velocity of the moving body. If the displacement $r_{s_{\mathrm{echo}} / b}^{b}$ between $b$ and $s_{\text {echo }}$ is small, then the lever arm effects can be neglected. If not, then it is recommended to introduce $\omega_{b / e, k}^{b}$ as a state in the system model as discussed in Section 4.4.1 and 5.3.1. Using the direct input method as discussed in Section 4.4 .2 results in a dynamic model and a sensor model that both uses the same IMU measurement as input. This introduces correlation between the disturbances 


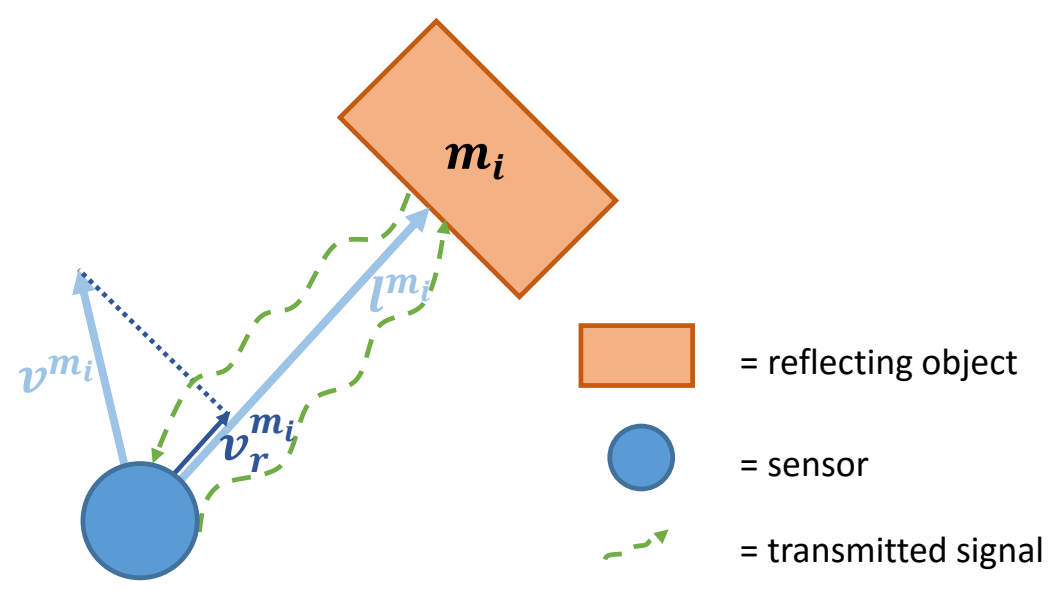

Figure 5.5: Illustration of the principle of reflected signal sensors. Information about the distance, $\left\|l^{m_{i}}\right\|_{2}$, and the radial velocity, $v_{r}^{m_{i}}$ can be obtained from the Doppler shift and time delay effects on the reflected signal.

in the system model and the measurement errors in the sensor model.

Note the extra time index $k$ on $l^{m_{i}}$ and $v^{m_{i}}$ introduced in (5.21a) and (5.21b) because of the introduced relations to the time-varying system states.

A measurement model that can be used for tracking can now be formed

$$
y_{i, k}^{\text {echo }}=\left(\begin{array}{c}
\left\|l^{m_{i}}\right\|_{2} \\
v^{m_{i}} \cdot \frac{l^{m_{i}}}{\left\|l^{m_{i}}\right\|_{2}}
\end{array}\right)+e_{i, k}^{\text {echo }},
$$

where $e_{i, k}^{\text {echo }}$ corresponds to the measurement errors and $l^{m_{i}}$ and $v_{k}^{m_{i}}$ are defined in (5.21a) and (5.21b). Note that $R_{s_{\text {echo }} / b}$ is not present in the model and hence do not need to be calibrated.

The positions $p_{m_{i} / e}^{e}$ of the reflecting objects in (5.21a) can be treated in different ways. They can be treated as known parameters, in which case they have to be mapped beforehand similar to $r_{s_{\text {echo }}^{b} b}^{b}$. They can also be treated as unknown states in an augmented system state vector, in which case they are estimated in a SLAM sense as described in Section 2.5.

\subsubsection{Model errors}

Just as for the camera and image feature case described in Section 5.4, it is difficult to say anything general about the measurement error $e_{i, k}^{\text {echo }}$ due to the existing variety of different signal processing methods available for extracting the distance and velocity information. However, it is clear that multipath and nonline-of-sight conditions will have a considerable impact on the errors just as for the UWB measurements described in Section 5.5. 
In Paper $\mathrm{C}$, the model (5.18) is re-parametrized into range and velocity parameters rather than the more commonly used Doppler shift and time delay parameters. This is because distance and velocity are more natural parameters for localization and tracking purposes. The paper studies the errors in range and velocity measurements as obtained from sound echoes, both theoretically using the CRLB and experimentally. The intention is to get better understanding of how a measurement and measurement error model should be defined. 



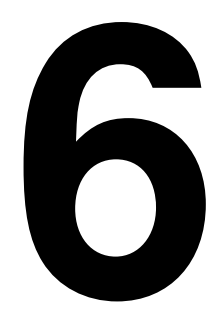

\section{Concluding remarks}

This thesis has introduced several different applications in which accurate tracking of pose in room-scaled environments is important. The focus for this thesis has been an application to metrology, the Virtual Photo Studio (VPS) project. The VPS project has a need for an accurate pose tracking system that can easily be moved between different photo studios. Such a tracking system enables the construction of the photometric models used in for scene reconstruction and image synthesis. To provide methods and algorithms to build a tracking system that satisfies the requirements of the VPS project has therefore been the focus of this thesis.

The Bayesian filtering framework and related Kalman filter algorithms was introduced as a solution to the problem of tracking pose. The framework allows for the same treatment of measurements from any type of sensor as long as it can be mathematically modeled. This enables fusion of sensors with complementary properties to increase tracking accuracy and robustness. Models of four different types of sensor measurements are given in the thesis. These sensors are used in Paper A-C where both theoretical, and experimental results for pose tracking in room-scaled environments are presented.

A first approach to pose tracking in this thesis was an inertial-camera filter using an external and known infrastructure of fiducial markers. The simulation results presented in Paper A are promising, showing that it is possible to reach the accuracy needed in the VPS project. The paper provides requirements on the accuracy of any image processing algorithm used to detect and identify the markers. Intuition about how the external infrastructure of fiducial markers should be deployed to get as much information as possible out of the camera measurements is also given. However, it is pointed out that the required number of markers to 
reach the VPS accuracy is high. This implies the need for an extensive external infrastructure and restricts the mobility of the tracking system. Also, since the purpose is to be able to build a photometric model of the environment this many markers will hide a large part of what needs to be visible.

Simultaneous localization and mapping (SLAM) was in this thesis used as a solution to the problem with the large external infrastructure of fiducial markers needed in Paper A. An inertial-camera SLAM algorithm is presented in Paper B, where naturally occurring landmarks are used rather than known markers. Even though all these three sensors are commonly used, the combination of them is novel. The presented algorithms is in the paper evaluated on real data. The results show that error drift is introduced when losing all information about the absolute world relying only on measurements of relative motion. This problem is however solved in the same paper by using time-of-flight measurements from a small number of easy-to-hide ultra wide band (UWB) transmitters. The solution presented in Paper $B$ is not completely self-sustainable but it is shown that as few as two UWB transmitters can eliminate the error drift in the experiments presented in Paper B. This drastically reduces the required external infrastructure compared to Paper A.

As mentioned before, the simulation study done on an inertial-camera tracking filter in Paper A shows promising results. However, the accuracy of the inertialcamera-UWB solution in Paper B do not fulfill the accuracy requirements of the VPS project. It is difficult to analyze if this is due to the change from known to naturally occurring landmarks, or because the quality of the inertial measurement unit and image processing algorithm used in the real experiment do not have the same accuracy as in the simulation study. It would be informative to do a simulation study also for the inertial-camera-UWB system to better pinpoint the required accuracy of the respective sensors. This would give important information about if and how VPS accuracy can be obtained also with the SLAM solution presented in Paper B.

The number of landmarks, and hence the estimated state vector and covariance matrix, can grow over time in a SLAM filter. For real-time performance it is therefore important to have an adaptive memory. The Kalman filter gain gives information about how much a certain measurement will effect the final filtered state estimate. To explore how this information can be used for an adaptive memory to determine which landmarks to keep and which to throw away could be an interesting extension of Paper B.

Both Paper A and B makes efforts to study the deployment of the external infrastructure used. This thesis suggest that the Cramér-Rao lower bound (CRLB) can be used as an important tool for analyzing and evaluation tracking scenarios and infrastructure configurations. To formalize this better, and explore methods for optimal deployment given different tracking scenarios, could improve tracking accuracy and help to make sure that the needed infrastructure is kept as small as possible.

To be able to build a high accuracy tracking system that, to a large degree, is self 
sustaining, it is important to use available measurements in a way such that no useful information is neglected. In this thesis it was proposed to use the CRLB as a tool also for analyzing raw measurements and signal processing methods to understand how to better use sensor and measurement error models. As an example, measurements from radio, light or sound sensors are, for the small scale environments studied in Paper $\mathrm{C}$, often used to obtain only range information. There is however a theoretical possibility to extract also velocity information from the Doppler effect, as done in larger scale tracking with for example radars and sonars. Paper C studies the CRLB of range and velocity estimates obtained from reflected known signals trough. It is shown that such range-velocity measurements have a strong correlation that is often neglected in tracking solutions. This is important information that can be used to improve tracking accuracy. Note that this result is also applicable to other scenarios than pose tracking in room-scaled environments, for example large-scale tracking with radar or sonar.

Measurements from a camera, obtained by extensive image processing and feature extraction, is another example of measurement that might not be fully exploited. Image processing methods most often provide only point measurements. The accuracy of these measurements, and any correlation between measurements obtained from the same image frame, are often not provided. This makes it difficult to know how to use these measurements in the best way possible. Based on the work in this thesis we see a possibility to, similarly to Paper C, use the CRLB to analyze image processing methods. This has the potential to provide important information, for example about how to design fiducial markers in order to make them detectable with high accuracy, or how to choose filter parameters in order to make use of the camera measurements in the best possible way. 



\section{Bibliography}

[1] O. 3.1.0. Camera calibration and $3 \mathrm{~d}$ reconstruction. http:// docs.opencv.org/3.1.0/d9/d0c/group_calib3d.html, September 2016.

[2] M. A. Al-Ammar, S. Alhadhrami, A. Al-Salman, A. Alarifi, H. S. Al-Khalifa, A. Alnafessah, and M. Alsaleh. Comparative survey of indoor positioning technologies, techniques, and algorithms. In 2014 International Conference on Cyberworlds (CW), pages 245-252, October 2014.

[3] N. A. Alsindi, B. Alavi, and K. Pahlavan. Measurement and modeling of ultrawideband TOA-based ranging in indoor multipath environments. IEEE Transactions on Vehicular Technology, 58(3):1046-1058, 2009.

[4] C. Andrieu and A. Doucet. Particle filtering for partially observed Gaussian state space models. Journal of the Royal Statistical Society: Series B (Statistical Methodology), 64(4):827-836, 2002.

[5] M. Athans, R. Wishner, and A. Bertolini. Suboptimal state estimation for continuous-time nonlinear systems from discrete noisy measurements. IEEE Transactions on Automatic Control, 13(5):504-514, October 1968.

[6] G. A. Aydemir and A. Saranl. Characterization and calibration of MEMS inertial sensors for state and parameter estimation applications. Measurement, 45(5):1210-1225, 2012.

[7] T. Bailey and H. Durrant-Whyte. Simultaneous localization and mapping (SLAM): part II. IEEE Robotics Automation Magazine, 13(3):108-117, June 2006.

[8] Y. Bar-Shalom, X. R. Li, and T. Kirubarajan. Estimation with applications to tracking and navigation: theory algorithms and software. John Wiley \& Sons, 2004.

[9] H. Bay, T. Tuytelaars, and L. Van Gool. SURF: Speeded up robust features. In European Conference on Computer Vision, pages 404-417, May 2006. 
[10] N. Bergman. Recursive Bayesian Estimation: Navigation and Tracking Applications. Dissertations no 579, Linköping Studies in Science and Technology, SE-581 83 Linköping, Sweden, May 1999.

[11] BeSpoon. Two way ranging. http://bespoon.com/ two-way-ranging/, September 2016.

[12] C. Bishop. Pattern Recognition and Machine Learning (Information Science and Statistics). Springer, New York, 2007.

[13] S. S. Blackman. Multiple hypothesis tracking for multiple target tracking. IEEE Aerospace and Electronic Systems Magazine, 19(1):5-18, January 2004.

[14] J.-Y. Bougue. Camera calibration toolbox for matlab. http://www . vision.caltech.edu/bouguetj/calib_doc/, September 2016.

[15] J. C. Butcher. Numerical methods for ordinary differential equations. John Wiley \& Sons, 2008.

[16] R. Campa and H. de la Torre. Pose control of robot manipulators using different orientation representations: A comparative review. In 2009 American Control Conference, pages 2855-2860, June 2009.

[17] D. Cassioli, M. Z. Win, and A. F. Molisch. The ultra-wide bandwidth indoor channel: From statistical model to simulations. IEEE Journal on Selected Areas in Communications, 20(6):1247-1257, August 2002.

[18] Y. Cho, J. Lee, and U. Neumann. A multi-ring color fiducial system and an intensity-invariant detection method for scalable fiducial-tracking augmented reality. In Proceedings of the First IEEE International Workshop on Augmented Reality (IWAR), pages 147-165, November 1998.

[19] T. A. Clarke and J. G. Fryer. The development of camera calibration methods and models. The Photogrammetric Record, 16(91):51-66, 1998.

[20] A. I. Comport, E. Marchand, and F. Chaumette. A real-time tracker for markerless augmented reality. In Proceedings of the 2nd IEEE and ACM International Symposium on Mixed and Augmented Reality (ISMAR), October 2003.

[21] J. M. Cooke, M. J. Zyda, D. R. Pratt, and R. B. McGhee. NPSNET: Flight simulation dynamic modeling using quaternions. Presence: Teleoperators \& Virtual Environments, 1(4):404-420, 1992.

[22] D. Dardari, A. Conti, U. Ferner, A. Giorgetti, and M. Z. Win. Ranging with ultrawide bandwidth signals in multipath environments. Proceedings of the IEEE, 97(2):404-426, 2009.

[23] P. Del Moral. Non-linear filtering: interacting particle resolution. Markov processes and related fields, 2(4):555-581, 1996. 
[24] T. Drummond and R. Cipolla. Real-time visual tracking of complex structures. IEEE Transactions on Pattern Analysis and Machine Intelligence (TPAMI), 24(7):932-946, July 2002.

[25] B. Duane C. Close-range camera calibration. Photogrammetic Engineering and Remote Sensing, 37(8):855-866, 1971.

[26] H. Durrant-Whyte and T. Bailey. Simultaneous localization and mapping: part I. IEEE Robotics Automation Magazine, 13(2):99-110, June 2006.

[27] H. Durrant-Whyte, D. Rye, and E. Nebot. Localization of Autonomous Guided Vehicles, pages 613-625. Springer London, 1996.

[28] N. El-Sheimy, H. Hou, and X. Niu. Analysis and modeling of inertial sensors using allan variance. IEEE Transactions on Instrumentation and Measurement, 57(1):140-149, January 2008.

[29] S. Eve. Augmenting phenomenology: Using augmented reality to aid archaeological phenomenology in the landscape. Journal of Archaeological Method and Theory, 19(4):582-600, 2012.

[30] M. Fiala. Artag, an improved marker system based on artoolkit. National Research Council Canada, Publication Number: NRC47419, 2004.

[31] M. A. Fischler and R. C. Bolles. Random sample consensus: A paradigm for model fitting with applications to image analysis and automated cartography. Communications of the ACM, 24(6):381-395, 1981.

[32] R. A. Fisher. On the mathematical foundations of theoretical statistics. The Philosophical Transactions of the Royal Society of London. Series A, Containing Papers of a Mathematical or Physical Character, A(222):309-368, 1922.

[33] R. A. Fisher. Theory of statistical estimation. In Mathematical Proceedings of the Cambridge Philosophical Society, volume 22, pages 700-725, 1925.

[34] W. T. Fong, S. K. Ong, and A. Y. C. Nee. Methods for in-field user calibration of an inertial measurement unit without external equipment. Measurement Science and Technology, 19(8), 2008.

[35] V. Fox, J. Hightower, L. Liao, D. Schulz, and G. Borriello. Bayesian filtering for location estimation. IEEE Pervasive Computing, 2(3):24-33, July 2003.

[36] P. Friend. Augmented reality implement control, December 2014. URL https://www.google.com/patents/US8918246. US Patent $8,918,246$.

[37] C. Fritsche, U. Orguner, and F. Gustafsson. On parametric lower bounds for discrete-time filtering. In 2016 IEEE International Conference on Acoustics, Speech and Signal Processing (ICASSP), pages 4338-4342, March 2016. 
[38] P. J. From and J. T. Gravdahl. Representing attitudes as sets of frames. In 2007 American Control Conference, pages 2465-2472, July 2007.

[39] J. G. Fryer and D. C. Brown. Lens distortion for close-range photogrammetry. Photogrammetric Engineering and Remote Sensing, 52(1):51-58, 1986.

[40] A. G. Gallagher, E. M. Ritter, H. Champion, G. Higgins, M. P. Fried, G. Moses, C. D. Smith, and R. M. Satava. Virtual reality simulation for the operating room: proficiency-based training as a paradigm shift in surgical skills training. Annals of surgery, 241(2):364-372, 2005.

[41] S. Gezici, Z. Tian, G. B. Giannakis, H. Kobayashi, A. F. Molisch, H. V. Poor, and Z. Sahinoglu. Localization via ultra-wideband radios: A look at positioning aspects for future sensor networks. IEEE Signal Processing Magazine, 22(4):70-84, July 2005.

[42] Z. Ghahramani. An introduction to hidden markov models and bayesian networks. International Journal of Pattern Recognition and Artificial Intelligence, 15(1):9-42, 2001.

[43] S. S. Goh, T. N. T. Goodman, and F. Shang. Joint estimation of time delay and doppler shift for band-limited signals. IEEE Transactions on Signal Processing, 58(9):4583-4594, September 2010.

[44] A. M. J. Goiser and G. L. Berger. Synchronizing a digital GPS receiver. In 8th Mediterranean Electrotechnical Conference (MELECON), volume 2, pages 1043-1046, May 1996.

[45] B. Graf. Quaternions and dynamics. arXiv preprint arXiv:0811.2889, 2008.

[46] M. Grewal and A. Andrews. Applications of Kalman filtering in aerospace 1960 to the present (historical perspectives). IEEE Control Systems, 30(3): 69-78, June 2010.

[47] M. Gruber. An approach to target tracking. Technical report, DTIC Document, 1967.

[48] J. E. Guivant and E. M. Nebot. Optimization of the simultaneous localization and map-building algorithm for real-time implementation. IEEE Transactions on Robotics and Automation, 17(3):242-257, June 2001.

[49] F. Gustafsson. Statistical sensor fusion. Studentlitteratur, 2010.

[50] F. Gustafsson. Particle filter theory and practice with positioning applications. IEEE Aerospace and Electronic Systems Magazine, 25(7):53-82, July 2010.

[51] F. Gustafsson and G. Hendeby. On nonlinear transformations of stochastic variables and its application to nonlinear filtering. In 2008 IEEE International Conference on Acoustics, Speech and Signal Processing, pages 3617-3620, April 2008. 
[52] F. Gustafsson and G. Hendeby. Some relations between extended and unscented Kalman filters. IEEE Transactions on Signal Processing, 60(2):545555, February 2012.

[53] P. Hämäläinen, T. Ilmonen, J. Höysniemi, M. Lindholm, and A. Nykänen. Martial arts in artificial reality. In Proceedings of the SIGCHI conference on Human factors in computing systems, pages 781-790, April 2005.

[54] C. Harris and M. Stephens. A combined corner and edge detector. In Alvey vision conference, volume 15, page 50, September 1988.

[55] S. Hashimoto, A. Ishida, M. Inami, and T. Igarashi. Touchme: An augmented reality based remote robot manipulation. In Proceedings of the 21st International Conference on Artificial Reality and Telexistence (ICAT), November 2011.

[56] J. Heikkila and O. Silvén. A four-step camera calibration procedure with implicit image correction. In Proceedings of the 1997 IEEE Computer Society Conference on Computer Vision and Pattern Recognition, pages 11061112, June 1997.

[57] J. P. Helferty and D. R. Mudgett. Optimal observer trajectories for bearings only tracking by minimizing the trace of the Cramér-Rao lower bound. In Proceedings of the 32nd IEEE Conference on Decision and Control, volume 1, pages 936-939, December 1993.

[58] G. Hendeby. Performance and Implementation Aspects of Nonlinear Filtering. Dissertations no 1161, Linköping Studies in Science and Technology, March 2008.

[59] M. L. Hernandez, T. Kirubarajan, and Y. Bar-Shalom. Efficient multisensor resource management using Cramér-Rao lower bounds. In Proceedings of SPIE, volume 4728, pages 394-409, August 2002.

[60] Y. Ho and R. Lee. A Bayesian approach to problems in stochastic estimation and control. IEEE Transactions on Automatic Control, 9(4):333-339, October 1964.

[61] H. G. Hoffman, D. R. Patterson, G. J. Carrougher, and S. R. Sharar. Effectiveness of virtual reality-based pain control with multiple treatments. The Clinical Journal of Pain, 17(3):229-235, 2001.

[62] J. D. Hol. Sensor Fusion and Calibration of Inertial Sensors, Vision, UltraWideband and GPS. Dissertations no 1368, Linköping Studies in Science and Technology, June 2011.

[63] Z. Hu and K. Uchimura. Real-time data fusion on tracking camera pose for direct visual guidance. In 2004 IEEE Intelligent Vehicles Symposium, pages 842-847, June 2004.

[64] J. C. Hung, J. R. Thacher, and H. V. White. Calibration of accelerometer triad of an IMU with drifting $\mathrm{z}$-accelerometer bias. In Proceedings of the 
IEEE 1989 National Aerospace and Electronics Conference (NAECON), volume 1, pages 153-158, May 1989.

[65] D. Jack, R. Boian, A. S. Merians, M. Tremaine, G. C. Burdea, S. V. Adamovich, M. Recce, and H. Poizner. Virtual reality-enhanced stroke rehabilitation. IEEE Transactions on Neural Systems and Rehabilitation Engineering, 9(3):308-318, 2001.

[66] S. Jayaram, J. Vance, R. Gadh, U. Jayaram, and H. Srinivasan. Assessment of VR technology and its applications to engineering problems. Journal of Computing and Information Science in Engineering, 1(1):72-83, 2001.

[67] A. H. Jazwinski. Stochastic Processes and Filtering Theory, volume 64 of Mathematics in Science and Engineering. Academic Press, INC., 1970.

[68] Q. Jin, K. M. Wong, and Z.-Q. Luo. The estimation of time delay and doppler stretch of wideband signals. IEEE Transactions on Signal Processing, 43(4):904-916, April 1995.

[69] R. E. Kalman. A new approach to linear filtering and prediction problems. Transactions of the ASME-Journal of Basic Engineering, 82(Series D):3545, 1960.

[70] R. E. Kalman and R. S. Bucy. New results in linear filtering and prediction theory. Journal of Fluids Engineering, 83(1):95-108, 1961.

[71] V. R. Kamat and S. El-Tawil. Evaluation of augmented reality for rapid assessment of earthquake-induced building damage. Journal of Computing in Civil Engineering, 21(5):303-310, 2007.

[72] M. Kanbara, H. Fujii, H. Takemura, and N. Yokoya. A stereo vision-based augmented reality system with an inertial sensor. In Proceedings of the IEEE and ACM International Symposium on Augmented Reality,(ISAR), pages 97-100, October 2000.

[73] H. Kato and M. Billinghurst. Marker tracking and hmd calibration for a video-based augmented reality conferencing system. In Proceedings of the 2nd IEEE and ACM International Workshop on Augmented Reality (IWAR), pages 85-94, October 1999.

[74] T. H. Kerr. Status of CR-like lower bounds for nonlinear filtering. IEEE Transactions on Aerospace and Electronic Systems, 25(5):590-601, Sepember 1989.

[75] M. Kok, N. Wahlström, T. B. Schön, and F. Gustafsson. MEMS-based inertial navigation based on a magnetic field map. In 2013 IEEE International Conference on Acoustics, Speech and Signal Processing, pages 6466-6470, May 2013.

[76] J. B. Kuipers et al. Quaternions and Rotation Sequences, volume 66. Princeton university press Princeton, 1999. 
[77] P. Lang, A. Kusej, A. Pinz, and G. Brasseur. Inertial tracking for mobile augmented reality. In Proceedings of the 19th IEEE Instrumentation and Measurement Technology Conference (IMTC), volume 2, pages 1583-1587, May 2002.

[78] S. L. Lauritzen. Time series analysis in 1880: A discussion of contributions made by T.N. Thiele. International Statistical Review / Revue Internationale de Statistique, 49(3):319-331, 1981.

[79] G. A. Lee, A. Dünser, S. Kim, and M. Billinghurst. Cityviewar: A mobile outdoor ar application for city visualization. In 2012 IEEE International Symposium on Mixed and Augmented Reality - Arts, Media, and Humanities (ISMAR-AMH), pages 57-64, November 2012.

[80] E. L. Lehmann. Theory of point estimation. John Wiley \& Sons, 1983.

[81] J. J. Leonard and H. J. S. Feder. A computationally efficient method for large-scale concurrent mapping and localization. In The Ninth Internatinal Symposium Robotics Research, pages 169-176, October 1999.

[82] X. R. Li and V. P. Jilkov. Survey of maneuvering target tracking. part i. dynamic models. IEEE Transactions on Aerospace and Electronic Systems, 39(4):1333-1364, October 2003.

[83] H. Liu, H. Darabi, P. Banerjee, and J. Liu. Survey of wireless indoor positioning techniques and systems. IEEE Transactions on Systems, Man, and Cybernetics, Part C (Applications and Reviews), 37(6):1067-1080, November 2007.

[84] M. A. Livingston, L. J. Rosenblum, D. G. Brown, G. S. Schmidt, S. J. Julier, Y. Baillot, J. E. Swan, Z. Ai, and P. Maassel. Military Applications of Augmented Reality, pages 671-706. Springer New York, 2011.

[85] D. G. Lowe. Distinctive image features from scale-invariant keypoints. International Journal of Computer Vision, 60(2):91-110, 2004.

[86] P. Maier, M. Tönnis, and G. Klinker. Augmented reality for teaching spatial relations. In Conference of the International Journal of Arts and Sciences, Toronto, May 2009.

[87] J. A. Marvel, J. Falco, and T. Hong. Ground truth for evaluating 6 degrees of freedom pose estimation systems. In Proceedings of the Workshop on Performance Metrics for Intelligent Systems, pages 69-74, March 2012.

[88] L. A. McGee and S. F. Schmidt. Discovery of the Kalman filter as a practical tool for aerospace and industry. Technical Report NASA Technical Memorandum 86847, NASA, 1985.

[89] M. McRae. Augmented reality remote control, June 2016. URL https://www.google.com/patents/US20160165170. US Patent App. 14/559,513. 
[90] J. L. Meriam and L. G. Kraige. Engineering mechanics: dynamics, volume 2. John Wiley \& Sons, 2012.

[91] P. Milgram, A. Rastogi, and J. J. Grodski. Telerobotic control using augmented reality. In 4th IEEE International Workshop on Robot and Human Communication, pages 21-29, July 1995.

[92] R. K. Miyake, H. D. Zeman, F. H. Duarte, R. Kikuchi, E. Ramacciotti, G. Lovhoiden, and C. Vrancken. Vein imaging: A new method of near infrared imaging, where a processed image is projected onto the skin for the enhancement of vein treatment. Dermatologic Surgery, 32(8):1031-1038, 2006.

[93] M. Montemerlo, S. Thrun, D. Koller, B. Wegbreit, et al. FastSLAM: A factored solution to the simultaneous localization and mapping problem. In Aaai/iaai, pages 593-598, 2002.

[94] M. Montemerlo, S. Thrun, D. Koller, and B. Wegbreit. FastSLAM 2.0: An improved particle filtering algorithm for simultaneous localization and mapping that provably converges. In Proceedings of the Sixteenth International Joint Conference on Artificial Intelligence (IJCAI), Acapulco, Mexico, 2003.

[95] A. Nehorai and M. Hawkes. Performance bounds for estimating vector systems. IEEE Transactions on Signal Processing, 48(6):1737-1749, June 2000 .

[96] U. Neumann and S. You. Natural feature tracking for augmented reality. IEEE Transactions on Multimedia, 1(1):53-64, March 1999.

[97] X. X. Niu, P. C. Ching, and Y. T. Chan. Wavelet based approach for joint time delay and doppler stretch measurements. IEEE Transactions on Aerospace and Electronic Systems, 35(3):1111-1119, July 1999.

[98] S. Noelle. Stereo augmentation of simulation results on a projection wall by combining two basic ARVIKA systems. In Proceedings of the International Symposium on Mixed and Augmented Reality (ISMAR), pages 271-322, September 2002.

[99] C. Norling and J. Österman. Physics Handbook for Science and Engineering. Studentlitteratur, 2002.

[100] H. Nurminen, T. Ardeshiri, R. Piche, and F. Gustafsson. Robust inference for state-space models with skewed measurement noise. IEEE Signal Processing Letters, 22(11):1898-1902, 2015.

[101] H. Nyqvist and F. Gustafsson. A high-performance tracking system based on camera and IMU. In 16th International Conference on Information Fusion (FUSION), pages 2065-2072, July 2013.

[102] H. E. Nyqvist, M. A. Skoglund, G. Hendeby, and F. Gustafsson. Pose estimation using monocular vision and inertial sensors aided with ultra wide 
band. In 2015 International Conference on Indoor Positioning and Indoor Navigation (IPIN), pages 1-10, October 2015. Runner up for the best paper award.

[103] H. E. Nyqvist, G. Hendeby, and F. Gustafsson. On joint range and velocity estimation in detection and ranging sensors. In 19th International Conference on Information Fusion (FUSION), pages 1674-1681, July 2016.

[104] U. Orguner and F. Gustafsson. Statistical characteristics of harris corner detector. In Proceedings of IEEE/SP 14th Workshop on Statistical Signal Processing, volume 2629, pages 571-575, August 2007.

[105] G. Papagiannakis, S. Schertenleib, B. O'Kennedy, M. Arevalo-Poizat, N. Magnenat-Thalmann, A. Stoddart, and D. Thalmann. Mixing virtual and real scenes in the site of ancient pompeii. Computer Animation and Virtual Worlds, 16(1):11-24, 2005.

[106] J. Park, B. Jiang, and U. Neumann. Vision-based pose computation: robust and accurate augmented reality tracking. In Proceedings of the 2 nd IEEE and ACM International Workshop on Augmented Reality (IWAR), pages 3-12, October 1999.

[107] C. J. Paulus, N. Haouchine, D. Cazier, and S. Cotin. Augmented reality during cutting and tearing of deformable objects. In 2015 IEEE International Symposium on Mixed and Augmented Reality (ISMAR), pages 5459, September 2015.

[108] J. Peng, Y. Ma, G. Gong, and L. Han. Real-time detection performance of airborne pulse doppler radar. In 2009 International Conference on Information Engineering and Computer Science, pages 1-4, December 2009.

[109] L. Porzi, E. Ricci, T. A. Ciarfuglia, and M. Zanin. Visual-inertial tracking on android for augmented reality applications. In 2012 IEEE Workshop on Environmental Energy and Structural Monitoring Systems (EESMS), pages 35-41, September 2012.

[110] G. Reitmayr and T. Drummond. Going out: Robust model-based tracking for outdoor augmented reality. In Proceedings of the 5th IEEE and ACM International Symposium on Mixed and Augmented Reality (ISMAR), pages 109-118, October 2006.

[111] B. Ristic, S. Arulampalam, and N. Gordon. Beyond the Kalman filter: Particle filters for tracking applications. Artech house Boston, 2004.

[112] S. Roweis and Z. Ghahramani. A unifying review of linear Gaussian models. Neural Computation, 11(2):305-345, February 1999.

[113] S. Särkkä. Bayesian filtering and smoothing, volume 3. Cambridge University Press, 2013.

[114] F. Seco, A. Jimenez, C. Prieto, J. Roa, and K. Koutsou. A survey of mathemat- 
ical methods for indoor localization. Intelligent Signal Processing, pages 9-14, 2009.

[115] M. P. Shutzenberger. A generalization of the Fréchet-Cramér inequality in he case of bayes estimation. Bulletine of the American Matchematical Society, 63(142), 1957.

[116] I. Skog and P. Händel. Calibration of a MEMS inertial measurement unit. In XVIII IMEKO World Congress, pages 17-22, Sepember 2006.

[117] D. Stricker, G. Klinker, and D. Reiners. A fast and robust line-based optical tracker for augmented reality applications. In Proceedings of the International Workshop on Augmented Reality (IWAR), pages 129-145, Natick, MA, USA, 1998. A. K. Peters, Ltd.

[118] C. Sweeney, J. Flynn, B. Nuernberger, M. Turk, and T. Höllerer. Efficient computation of absolute pose for gravity-aware augmented reality. In 2015 IEEE International Symposium on Mixed and Augmented Reality (ISMAR), pages 19-24, September 2015.

[119] P. Swerling. Proposed Stagewise Differential Correction Procedure for Satellite Tracking and Prediction. P: Rand Corporation. Rand Corporation, 1958.

[120] P. Swerling. First Order Error Propagation in a Stagewise Smoothing Procedure for Satellite Observations. P: Rand Corporation. Rand Corporation, 1959.

[121] R. Szeliski. Computer vision: Algorithms and Applications. Springer Science \& Business Media, 2010.

[122] F. J. Taylor. Adaptive pulse doppler ambiguity resolution. IEEE Transactions on Aerospace Electronic Systems, 12:98-103, 1976.

[123] J. Taylor. The Cramér-Rao estimation error lower bound computation for deterministic nonlinear systems. IEEE Transactions on Automatic Control, 24(2):343-344, April 1979.

[124] P. Tichavsky, C. H. Muravchik, and A. Nehorai. Posterior Cramér-Rao bounds for discrete-time nonlinear filtering. IEEE Transactions on Signal Processing, 46(5):1386-1396, May 1998.

[125] D. Titterton and J. L. Weston. Strapdown Inertial Navigation Technology, volume 17. IET, 2004.

[126] L. University. The virtual photo set. http://www.itn.liu. se/mit/research/computer-graphics-image-processing/ virtual-photo-sets, October 2016.

[127] L. Vacchetti, V. Lepetit, and P. Fua. Combining edge and texture information for real-time accurate $3 \mathrm{~d}$ camera tracking. In Third IEEE and ACM In- 
ternational Symposium on Mixed and Augmented Reality (ISMAR), pages 48-56, November 2004.

[128] H. L. Van Trees. Detection, estimation, and modulation theory part 1. John Wiley \& Sons, 1968.

[129] H. L. Van Trees, K. L. Bell, and Z. Tian. Detection, estimation, and modulation theory Part 1. John Wiley \& Sons, second edition, 2013.

[130] S. Vogt, A. Khamene, F. Sauer, and H. Niemann. Single camera tracking of marker clusters: multiparameter cluster optimization and experimental verification. In Proceedings of the International Symposium on Mixed and Augmented Reality (ISMAR), pages 127-136, October 2002.

[131] E. A. Wan and R. V. D. Merwe. The unscented Kalman filter for nonlinear estimation. In The IEEE Adaptive Systems for Signal Processing, Communications, and Control Symposium, pages 153-158, October 2000.

[132] Z. Wang, L. Ng, S. Ong, and A. Nee. Assembly planning and evaluation in an augmented reality environment. International Journal of Production Research, 51(23-24):7388-7404, 2013.

[133] S. Weiss, M. W. Achtelik, S. Lynen, M. Chli, and R. Siegwart. Real-time onboard visual-inertial state estimation and self-calibration of MAVs in unknown environments. In 2012 IEEE International Conference on Robotics and Automation (ICRA), pages 957-964, May 2012.

[134] M. Z. Win and R. A. Scholtz. On the energy capture of ultrawide bandwidth signals in dense multipath environments. IEEE Communications Letters, 2(9):245-247, September 1998.

[135] O. J. Woodman. An introduction to inertial navigation. University of Cambridge, Computer Laboratory, Technical Report UCAMCL-TR-696, 2007.

[136] Y. Yang and A. E. Fathy. Development and implementation of a real-time see-through-wall radar system based on FPGA. IEEE Transactions on Geoscience and Remote Sensing, 47(5):1270-1280, 2009.

[137] K. B. Yesin and B. J. Nelson. A CAD model based tracking system for visually guided microassembly. Robotica, 23(04):409-418, 2005.

[138] X. Zhang, S. Fronz, and N. Navab. Visual marker detection and decoding in ar systems: a comparative study. In Proceedingsof the International Symposium on Mixed and Augmented Reality (ISMAR), pages 97-106, October 2002.

[139] Z. Zhang. A flexible new technique for camera calibration. IEEE Transactions on Pattern Analysis and Machine Intelligence, 22(11):1330-1334, November 2000.

[140] G. Zhao, L. Chen, J. Song, and G. Chen. Large head movement tracking us- 
ing SIFT-based registration. In Proceedings of the 15th ACM International Conference on Multimedia, pages 807-810, September 2007.

[141] F. Zhu, S. Gao, A. T. S. Ho, T. W. Brown, J. Li, and J.-D. Xu. Low-profile directional ultra-wideband antenna for see-through-wall imaging applications. Progress In Electromagnetics Research, 121:121-139, 2011.

[142] M. Zyda. From visual simulation to virtual reality to games. Computer, 38 (9):25-32, September 2005. 


\section{Part II}

\section{Publications}





\section{Publications}

The articles associated with this thesis have been removed for copyright reasons. For more details about these see:

$\underline{\text { http://urn.kb.se/resolve?urn=urn:nbn:se:liu:diva-132735 }}$ 